\title{
Anonymous Price Taking Equilibrium in Tiebout Economies With a CONTINUUm OF AgENTS; ExistenCE AND CHARACTERIZATION \\ by
}

Nizar Allouch, John P. Conley, and Myrna Wooders

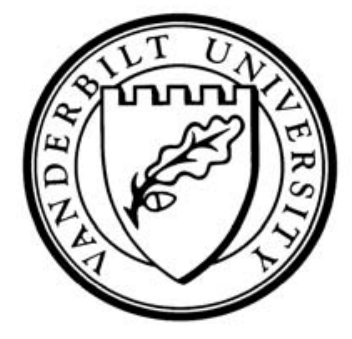

Working Paper No. 08-W11

June 2008

DEPARTMENT OF ECONOMICS

VANDERBILT UNIVERSITY

NASHVILLE, TN 37235

www.vanderbilt.edu/econ 


\title{
Anonymous Price Taking Equilibrium in Tiebout Economies with a Continuum of Agents; Existence and Characterization *
}

\author{
Nizar Allouch \\ Department of Economics, Queen Mary, University of London \\ n.allouch@qmul.ac.uk \\ John P. Conley and Myrna Wooders \\ Department of Economics, University of Vanderbilt \\ j.p.conley@vanderbilt.edu, myrna.wooders@vanderbilt.edu \\ url: myrnawooders.com \\ This version: June 2008. \\ Key words: Tiebout, jurisdictions, f-core, core-equilibrium \\ equivalence, Edgeworth equivalence, continuum economies, \\ crowding types, core, equal treatment core, large games \\ JEL Numbers: C7, D5, H4
}

${ }^{*}$ A version of this paper is to appear in Journal of Mathematical Economics. We are indebted to Manfredo Dix, Vladislav Gorlov, Mamoru Kaneko, Hideo Konishi, Tom Nechyba, and especially Jaime Luque for helpful comments. We are also indebted to the National Science Foundation of the U.S. and the Social Sciences and Humanities Research Council of Canada for support. We are pleased to submit this paper to a special issue of Journal of Mathematical Economics in honor of Aloisio Araujo. 


\begin{abstract}
We introduce a model of a local public goods economy with a continuum of agents and jurisdictions with finite, but unbounded populations, where the set of possible projects for each jurisdiction/club is unrestricted in size. Under boundedness of per capita payoffs, which simply ensures that equal treatment payoffs are bounded above, we apply results of Kaneko and Wooders (1986) to obtain nonemptiness of the core of the economy. We then demonstrate, under the stronger condition of strict small group effectiveness, that the equal treatment core coincides with the set of price-taking equilibrium outcomes with anonymous prices - that is, prices for public goods depend only on observable characteristics of agents. Existence of equilibrium follows from nonemptiness of the core and equivalence of the core to the set of equilibrium outcomes. Our approach provides a new technique for showing existence of equilibrium in economies with a continuum of agents.
\end{abstract}




\section{Introduction}

In his seminal paper, Tiebout (1956) suggested that if public goods are subject to congestion and exclusion - that is, if public goods are local rather than pure - then the benefits of sharing costs over a large number of consumers will eventually be offset by the negative effects of congestion. Balancing the effects of cost-sharing and congestion make it advantageous for consumers to be partitioned into a system of multiple disjoint jurisdictions. Tiebout speculated that, in such a situation, jurisdictions offer competing bundles of local public goods levels and tax liabilities and consumers locate in jurisdictions with public goods and taxes most closely approximating their ideal combinations. Tiebout concluded that if public goods are local, consumers will reveal their preferences through their locational choice and the well-known free rider problem will disappear.

In effect, Tiebout hypothesized that in a local public goods economy, competitive forces will lead to near efficient equilibrium outcomes. While he made an intriguing case for the preference revelation properties of equilibrium, Tiebout's paper is quite informal. Subsequent more rigorous investigations have shown that the nonexistence problem is far from trivial. One such investigation, Bewley (1981), presents a series of examples to show that competitive equilibrium may not exist and, when it does, equilibrium outcomes may not be efficient. While Bewley's counterexamples are not comprehensive (indeed, he does not recognize the importance of small group effectiveness, discussed below) his paper illustrates that adding jurisdictions, clubs, coalition production and/or consumption, and so on to an economy creates additional difficulties, distinct from those seen in standard private goods economies, in proving the existence of competitive equilibrium.

Not surprisingly, the question of existence of equilibrium in Tiebout economies, or economies with other sorts of collective activities subject to congestion, has occupied the attention of many authors. We divide their contributions into four branches and give a brief discussion below, thus providing a larger context for our contribution.

The first branch treats a model with a continuum of consumers who divide themselves into an exogenously fixed, finite number of jurisdictions, each of which chooses public goods levels according to a voting rule. All these papers consider Nash equilibrium and differ mainly in their treatments of land and taxation rules. Notable contributions include Westhoff (1977), Dunz (1989), Greenberg and Shitovitz (1988), Konishi (1996, 2006), Rose- 
Ackerman (1979), Epple, Filimom and Romer (1984, 1993), and Nechyba (1996). This literature succeeds in addressing the very difficult question of the existence of equilibrium in a variety of interesting institutional environments; these equilibria are not, in general, Pareto efficient so in the contexts of these papers Tiebout's hypothesis is unconfirmed. Note that a feature of these models is that there is a continuum of consumers divided into a fixed finite number of jurisdictions. If we interpret the continuum as an uncountable infinity of consumers, this means that almost all consumers live in jurisdictions with uncountably infinite populations. Note in particular that with a finite number of jurisdictions, at best only a zero measure of consumers could live in small (meaning finite) towns. Thus, by construction, small jurisdictions or clubs (arising from matching games, for example) are ruled out. We will return to this point below.

A second branch of the literature addresses some of the issues discussed above by considering the existence of the core or other, not-necessarily pricetaking, Pareto efficient equilibria in finite economies. Notable papers here include Wooders $(1978)^{1}$, Guesnerie and Oddou (1981), Weber and Zamir (1985), Greenberg and Weber (1986), Demange (1994), and Conley and Konishi (2002). The tenor of the results of this literature is that first best equilibrium can be shown to exist only under restrictive circumstances (e.g. the economy can be partitioned into groups of "type optimal jurisdictions" or the economy has three or fewer consumers or there is only one public good and consumers' preferences are identical and single-peaked.)

A third branch of the literature was initiated by Ellickson (1979), who treats local public goods as a fixed finite number of indivisible commodities. These indivisible public goods are closer in spirit to public services as described by Bewley (1981), for example, than to nonrival public goods. Vohra (1987) treats a similar model and shows the existence of second best approximate equilibrium while Vohra (1984) treats a continuum version of his model.

To summarize these first three literatures, except under special conditions or with market frictions, ${ }^{2}$ there is no general proof of nonemptiness of the core or of existence of Nash equilibrium in pure strategies or of efficient pricetaking equilibrium states of the economy. Thus, at this point we are left with

\footnotetext{
${ }^{1}$ We note that most of these papers require that there be only one private good for the results to hold.

${ }^{2}$ For situations with possible market frictions, see Wooders $(1988,1989)$ and Allouch and Wooders (2008).
} 
a choice between models for which equilibrium, if it exists, is efficient, but the conditions for existence appear quite restrictive and models for which equilibrium exists but is in general inefficient.

The final branch of the literature is relatively small but suggests a possible solution to these problems and motivates the approach taken in the current paper. This branch of the literature treats finite economies in which crowding or congestion limits the size of efficient or near-efficient jurisdictions to be small relative to the population. ${ }^{3}$ A basic insight of Pauly (1972) and Wooders (1978) is that what drives the nonexistence of the core and of competitive equilibrium in general is that the total number of consumers of each type may not be an exact multiple of the efficient size of a jurisdiction for that type. Pauly treats an economy as a transferable utility game where all consumers are essentially identical so that the optimality of a jurisdictional size does not depend on the types of consumers residing in the jurisdiction. Wooders (1978) shows that: unless consumers of different types have the same demands for local public goods and congestion, then states of the economy in the core and equilibrium states coincide and have homogeneous jurisdictions ${ }^{4}$ and; if it is assured that the population can be partitioned into type optimal jurisdictions $^{5}$, then the core is nonempty, equilibrium exists, and outcomes in the core and equilibrium outcomes are equivalent. Moreover, even if the population cannot be partitioned into type optimal jurisdictions, then, as the population grows, the proportion of 'left-overs' - consumers who cannot be accommodated in optimally sized groups for their types - goes to zero and existence of approximate equilibrium and nonemptiness of approximate cores obtain (Wooders 1980).

The research of Wooders $(1978,1980)$ treats economies with anonymous crowding; individuals are affected only by the numbers of individuals in the same jurisdiction and not their attributes. The basic results treating cores and equilibrium, however, hold in much broader contexts. Roughly, in application to economies with local public goods or clubs, subsequent papers

\footnotetext{
${ }^{3}$ See, in addition to the research cited above, McGuire (1974), Hamilton (1975), and Berglas and Pines (1981) who discuss optimal (rather than core) states of the economy.

${ }^{4}$ It is required that there be only one private good for these results to hold. As later shown, although the results stated for the one-private-good case are correct, the statement of existence of equilibrium with more than one private good in Wooders (1978) requires more conditions.

${ }^{5}$ Jurisdictions that are of optimal size from the viewpoint of their membership under the assumption of equal sharing of costs by members of the same jurisdiction
} 
show that, if almost all gains to collective activities can be realized by relatively small groups of consumers then approximate cores are nonempty and the approximation can be made arbitrarily close as the economies grow large (see Wooders 1983 for first results and Kovalenkov and Wooders 2003 for more recent results and discussion of the literature). Moreover, approximate equilibrium, or equilibrium with communication costs, exist and cores converge to equilibrium outcomes (see Wooders 1985, 1988, 1997 and Allouch and Wooders 2008). The main conditions of these papers, implying either small group effectiveness (that relatively small groups can realize almost all gains to group formation) or simply per capita boundedness (finiteness of the supremum of average feasible utility levels) are apparently quite nonrestrictive. In some cases, Wooders (1983) and Allouch and Wooders (2008), for example, just per capita boundedness suffices.

Two recent papers in this branch of the literature are Cole and Prescott (1997) and Ellickson et al. (1999). Cole and Prescott (1997) treats valuation equilibrium and resolves the existence problem through the use of lotteries over club memberships. Ellickson et al. (1999) study a local public goods economy similar to that of Conley and Wooders (1997) and Cole and Prescott (1997). By fixing a finite menu of admissible sorts of club types and allowing only a finite number of distinct public projects, thus uniformly bounding the size of admissible clubs and the sorts of clubs that are possible, Ellickson et al. are able to adopt techniques from finite-dimensional private goods exchange economies to prove existence of equilibrium and equivalence of outcomes in the core and equilibrium outcomes. As opposed to requiring that consumers join one and only one jurisdiction, as may be appropriate in the context of local public goods, Ellickson et al., (as Shubik and Wooders, 1982; Allouch and Wooders, 2008), allow consumers to join several clubs.

In this paper it is especially noteworthy that we do not limit the possible public projects to a pre-set finite list. Instead we allow projects to be drawn from a metric space and thus our framework accommodates local public goods as they are usually modeled. This allows us to consider, standard public goods (which are drawn from a convex Euclidian space) as special case. Second, we do not place an ex ante bound on jurisdictional size as a foundational assumption of the model. Instead we require only that jurisdictions be finite. In both these respects, our model differs from that of Ellickson et al. We argue from an economic standpoint it may not be reasonable to set an ex-ante bound on club size and that the space of public projects need not be limited to a finite set. This means we must invent a new approach to 
demonstrating existence of equilibrium.

Rather than restricting to a pre-set menu of clubs, (or jurisdictions) we allow consumers to form finite jurisdictions, unbounded in numbers of members. Following Kaneko and Wooders (1986), partitions of consumers into jurisdictions are required to be consistent with the proportions given by the measure on the set of consumers. We show the existence of Pareto efficient Tiebout equilibrium with a continuum of consumers. As we note above, a key aspect of our paper is that jurisdiction sizes are unbounded. An additional contribution of our paper is to introduce a more concise statement of measurement consistency.

Since the number of private goods may be greater than one, improving coalitions may form multiple jurisdictions and engage in coalition-wide trade in private goods. To obtain our results on the core, we adapt the $f$-core notion of Kaneko and Wooders $(1986,1989)$ and Hammond, Kaneko and Wooders (1989). This notion of the core requires that no finite coalition can improve. An alternative approach would be to use the Aumann notion of the core which requires that improving coalitions be of positive measure. Our model, however, encompasses situations such as matching models, where individuals form two-person partnerships or clubs. Moreover, in any of the models discussed in this branch of the literature, individuals are affected by the other members of the same jurisdiction; individuals care about the attributes of other individuals. In this context, it is natural to have coalitions consisting of finite sets of individual consumers. ${ }^{6}$

To summarize, in this paper we introduce a model of an economy with a continuum of consumers, multiple public and private goods, and finite but unbounded jurisdictions sizes. Following Conley and Wooders $(1996,1997,2001)$, we make a distinction between the unobservable taste types of consumers and their observable crowding types. The crowding type of a consumer determines his effects on other consumers and/or on production possibilities. We define a competitive equilibrium concept in which admission prices for

\footnotetext{
${ }^{6}$ Kaneko and Wooders (1986) show that, in the context of private goods economies without externalities, the notion of the core with coalitions of positive measure and the $f$-core coincide. In our model, since all "externalities" are within jurisdictions - sets of measure zero - it would be possible to use the notion of the core with coalitions of positive measure, but in view of the considerations above, we choose the $f$-core. We also remark that the $f$-core and the core of a game with a finite number of consumers are determined by the same set of axioms (Winter and Wooders, 1994), providing further justification of the $f$-core notion.
} 
jurisdictions depend only on observable crowding types of consumers. Under apparently mild conditions we show that the core is nonempty. We also show that the set of core outcomes with the equal treatment property - outcomes which yield identical utilities to identical consumers - coincides with the set of equilibrium outcomes. Conditions are described under which all outcomes in the core have the equal treatment property and the core coincides with the equilibrium outcomes. Thus, from nonemptiness of the core and the equivalence of the core with the competitive outcomes, we obtain existence of Pareto-efficient competitive equilibrium. A discussion of the literature is provided in Section 3.

\section{The model and results}

Let $(N, \beta, \mu)$ be a measure space where $N$ (the set of consumers) is a Borel subset of a complete separable metric space, let $\beta$ be a $\sigma$-algebra of all Borel subsets of $N$ and let $\mu$ be a nonatomic measure with $0<\mu(N)<+\infty$. Each consumer $i \in N$ is endowed with one of $C$ different sorts of crowding types, ${ }^{7}$ denoted $c \in\{1, \ldots, C\} \stackrel{\text { def }}{=} \mathcal{C}$, and one of $T$ different sorts of taste types, denoted $t \in\{1, \ldots, T\} \stackrel{\text { def }}{=} \mathcal{T}$. An element of $\mathcal{C} \times \mathcal{T}$ is typically represented by a pair $(c, t)$ and is called a consumer's type. The assignment of crowding and taste types to individual consumers are given by a pair of attribute functions, denoted, respectively, by $\kappa: N \mapsto \mathcal{C}$ and $\tau: N \mapsto \mathcal{T}$. For each $(c, t)$, and for any measurable subset $S$ of $N$, we define

$$
\begin{aligned}
& S_{c t} \stackrel{\text { def }}{=}\{i \in S: \kappa(i)=c \text { and } \tau(i)=t\}, \\
& \text { the consumers of type }(c, t) \text { in } S .
\end{aligned}
$$

A jurisdiction $G$ is a finite subset of $N$. Let $\mathcal{F}$ be a given set of (admissible) jurisdictions. The set $\mathcal{F}$ is required to satisfy the property that for each $i \in N,\{i\} \in \mathcal{F}$. For example, the set $\mathcal{F}$ may be the set of all finite subsets of $N$, or it may be simply the set of all singleton subsets. In the following, whenever we refer to a jurisdiction, we mean an element of the set $\mathcal{F}$. Note that if $\mathcal{F}=\{\{i\} \in N\}$, then the economy will have, in effect, only private goods - that is, each person consumes his private goods, without any direct effects of other consumers. We observe that the structure can accommodate,

\footnotetext{
${ }^{7}$ It is easy to generalized this to a crowding type being a point in a finite or infinite dimensional space.
} 
as a special case, assignment or matching models since in these models one choice open to a consumer is to remain unmatched.

We describe a jurisdiction by the numbers of consumers of each type in the jurisdiction. Let $\mathbb{Z}$ denote the nonnegative integers and let $\mathbb{Z}^{C T}$ denote the $C T$-fold Cartesian product of $\mathbb{Z}$. Given an admissible jurisdiction $G \in \mathcal{F}$, the profile of $G$, denoted by $\operatorname{pro}(G)$, is a vector in $\mathbb{Z}^{C T}$ defined by its components

$$
\operatorname{pro}(G)_{c t} \stackrel{\text { def }}{=}\left|G_{c t}\right|
$$

where $|\cdot|$ denotes the cardinality of a set. The vector $\operatorname{pro}(G)$ describes the jurisdiction $G$ by the number of consumers of each type in the jurisdiction. The crowding profile of a jurisdiction $G$, denoted by $\mathcal{C} \operatorname{pro}(G)$, is a vector in $\mathbb{Z}^{C}$ defined by its components

$$
\mathcal{C} \operatorname{pro}(G)_{c} \stackrel{\text { def }}{=}\left|G_{c}\right| .
$$

One of the most crucial concepts in our work is that of a jurisdiction structure. Since consumers consume public goods jointly with other members of finite jurisdictions, a feasible state of the economy must specify a partition of the set of consumers into finite jurisdictions that is consistent with the measure on the total consumer set. Thus, a jurisdiction structure is a measurement-consistent partition in the sense of Kaneko and Wooders (1986). We provide here the definition.

Let $S$ be a measurable subset of $N$ and let $\pi$ denote a partition of $S$ into jurisdictions. The partition $\pi$ is measurement consistent if, for each positive integer $\nu$ it holds that

$S_{\nu}:=\bigcup_{G \in \pi,|G=\nu|} G$ is a measurable set and each $S_{\nu}$ has a partition into measurable subsets $\left\{S_{\nu 1}, \ldots, S_{\nu \nu}\right\}$ with the property that there are measurepreserving isomorphisms $\phi_{\nu 1}, \ldots, \phi_{\nu \nu}$ from $S_{\nu 1}$ to $S_{\nu 1}, \ldots, S_{\nu \nu}$ respectively where $\phi_{\nu 1}$ is the identity map and $\left\{\phi_{\nu 1}(i), \ldots, \phi_{\nu \nu}(i)\right\} \in \pi$ for all $i \in S_{\nu 1}$.

Informally, $S_{\nu}$ is the set of consumers who belong to $\nu$-member jurisdictions in the partition $\pi$. Measurement consistency requires that we can partition $S_{\nu}$ into $\nu$ subsets, $\left\{S_{\nu 1}, \ldots, S_{\nu \nu}\right\}$, so that each set $S_{\nu k}$ contains one and only one member of a jurisdiction. We can think of those consumers in $S_{\nu 1}$ as "first members" of jurisdiction in $\pi$ and, for each $k=1, \ldots, \nu$, as those consumers in $S_{\nu k}$ as " $k t h$ members of jurisdictions in $\pi$. Then, given $i \in S_{\nu}$, 
$\left\{\phi_{\nu 1}(i), \ldots, \phi_{\nu \nu}(i)\right\}$ lists the other members of the same jurisdiction as $i$. The feature that each $\phi_{\nu k}$ is measure-preserving rules of the sort of phenomena illustrated by the partition $\pi$ in Example 1 below.

The existence of a measurement consistent partition of a measurable set is demonstrated in Kaneko and Wooders (1986, Lemma A.2).

Example 1. Let $N=[0,3)$ be the set of consumers endowed with Lebesgue measure. The consumers in $[0,1)$ are girls and those in $[1,3)$ are boys. Intuitively, there are twice as many boys as girls. Let $\pi$ be a partition of the consumer set into boy-girl pairs given by

$$
\pi=\{(i, j): i \in[0,1), j=1+2 i\}
$$

that is, girl $i$ is partnered with boy $1+2 i$. Note that every boy has a partner! This is inconsistent with the intuition that there are twice as many boys as girls. And it is clear that $\pi$ is not measurement consistent. To see this, take the set of girls as the set of first members of matchings, that is, in the notation above, let $S_{\nu 1}=[0,1)$. For this example, measurement consistency requires the existence of a measure-preserving isomorphism from $[0,1)$ to $[1,3)$, which is impossible.

In contrast, let $\pi^{\prime}$ be the partition given by $\pi^{\prime}=\{(i, j): i \in[0,1), j=$ $1+i\} \cup\{j: j \in[2,3)\}$. This partition reflects the relative abundances given by the measure.

For simplicity we will often refer to a jurisdiction structure of $N$ as simply a jurisdiction structure. Given a particular jurisdiction structure $\pi$ and consumer $i \in N$, let $\pi_{i}$ denote the jurisdiction in $\pi$ containing consumer $i$.

We consider an economy with $L$ private goods and a metric space of public projects, denoted by $\mathcal{X}^{\text {public }}$, containing a distinguished element denoted by $\underline{0} .{ }^{8}$ A bundle of private goods is denoted by $x \in \mathbb{R}_{+}^{L}$ and a public project is denoted by $y \in \mathcal{X}^{\text {public }}$. An endowment is given by a measurable and integrable function $\omega^{0}$ from $N$ to $\mathbb{R}_{+}^{L}$ such that, for all consumers $i$ and $j$ with $\tau(i)=\tau(j)$, it holds that $\omega^{0}(i)=\omega^{0}(j)$.

Each consumer of type $(c, t) \in \mathcal{C} \times \mathcal{T}$ has a consumption set

$$
\mathcal{X}_{c t}=\mathbb{R}_{+}^{L} \times \mathcal{X}^{\text {public }} \times \mathbb{Z}_{c t},
$$

\footnotetext{
${ }^{8}$ Following Mas-Colell (1980) for economies with public projects, we do not require a linear structure on the space $\mathcal{X}^{\text {public }}$. This is costless - the proofs of our results are the same as in the case where $\mathcal{X}^{\text {public }}$ is contained in some finite dimensional Euclidean space.
} 
where $\mathbb{Z}_{c t} \subset \mathbb{Z}^{C T}$ is the set of profiles $\operatorname{pro}(S)$ with $\operatorname{pro}(S)_{c t} \neq 0$. (The motivation for the restriction of crowding profile for a consumer of type $(c, t)$ to $\mathbb{Z}_{c t}$ is clear - it is not possible for a consumer to belong to a jurisdiction that contains no consumers of his type.)

Note that, from our assumptions, a consumer can produce zero public projects while consuming his endowment in a jurisdiction consisting of himself alone. Note also that neither $\mathbb{R}_{+}^{L}$ nor $\mathcal{X}^{\text {public }}$ depend on consumers' types. A consumer's preferences, however, are only defined over those jurisdiction profiles containing consumers of his crowding type; if a consumer is of type $(c, t)$ and $(x, y, \operatorname{pro}(G))$ is in $\mathcal{X}_{c t}$, then $\operatorname{pro}(G)_{c t}$ is not equal to zero.

The preferences of a consumer of type $t$ are described by a continuous utility function $u_{t}$ mapping $\mathcal{X}_{c t}$ into $\mathbb{R}_{+}$. In interpretation,

$$
u_{t}(x, y, \operatorname{pro}(G))<u_{t}\left(x^{\prime}, y^{\prime}, \operatorname{pro}\left(G^{\prime}\right)\right)
$$

means that a consumer of taste type $t$, in a jurisdiction with profile $\operatorname{pro}\left(G^{\prime}\right)$, enjoys the bundle $\left(x^{\prime}, y^{\prime}\right)$ of private goods and public projects more than he would enjoy the bundle $(x, y)$ in a jurisdiction with profile $\operatorname{pro}(G)$. We assume that utility functions are continuous and strictly increasing in private goods consumption. ${ }^{9}$

Given $i \in N$ with $\tau(i)=t$, define

$$
u_{i}(x, y, \operatorname{pro}(G)) \stackrel{\text { def }}{=} u_{t}(x, y, \operatorname{pro}(G)) .
$$

For each taste type $t \in \mathcal{T}$ we make the following assumptions, dictating that preferences depend only on crowding characteristics of consumers in the same jurisdiction and not on their preferences:

(A.1) Taste anonymity in consumption (TAC): For all $x \in \mathbb{R}_{+}^{L}, y \in \mathcal{X}^{\text {public }}$ and all $G, G^{\prime} \in \mathcal{F}$ such that $(x, y, \operatorname{pro}(G)),\left(x, y, \operatorname{pro}\left(G^{\prime}\right)\right) \in \mathcal{X}_{c t}$ and $\mathcal{C}$ pro $(G)=\mathcal{C} p r o\left(G^{\prime}\right)$ it holds that $u_{t}(x, y, \operatorname{pro}(G))=u_{t}\left(x, y, \operatorname{pro}\left(G^{\prime}\right)\right)$.

\footnotetext{
${ }^{9}$ These are stronger assumptions than required - in fact, for private goods, we could use the assumptions of Hammond, Kaneko and Wooders (1989). However, we prefer to keep our paper more focused on the main issues of this paper rather than including generality that does not illuminate these issues.
} 
Production of the public good is also subject to crowding. ${ }^{10}$ We allow the production technology available to a jurisdiction to depend on the profile of the membership of the jurisdiction. Formally, the production technology is given by a mapping $P$ from the set of profiles to nonempty, closed subsets of $\mathbb{R}_{+}^{L} \times \mathcal{X}^{\text {public }}$ containing $(0, \underline{0}) .{ }^{11}$ Thus, for each jurisdiction $G$,

$$
\begin{aligned}
P(\operatorname{pro}(G)) & \subset \mathbb{R}_{+}^{L} \times \mathcal{X}^{\text {public }} \text { and } \\
(0, \underline{0}) & \in P(\operatorname{pro}(G))
\end{aligned}
$$

and $P(\operatorname{pro}(G))$ represents the technology for all jurisdictions with profile $\operatorname{pro}(G)$.

(A.2) Given a jurisdiction $G \in \mathcal{F}$ and a vector $\bar{z} \in \mathbb{R}_{+}^{L}$ the set $\{(z, y) \in$ $P($ pro $\left.(G)): z \in \mathbb{R}_{+}^{L}, z \leq \bar{z}\right\}$ is compact. ${ }^{12}$

For our price system to be Pareto-optimal, we require taste anonymity in production as well as in consumption.

(A.3) Taste anonymity in production (TAP): For all $G, G^{\prime} \in \mathcal{F}$ such that $\mathcal{C}$ pro $(G)=\mathcal{C}$ pro $\left(G^{\prime}\right)$ it holds that $P(\operatorname{pro}(G))=P\left(\operatorname{pro}\left(G^{\prime}\right)\right)$.

To define feasible states of the economy, we require that any feasible state is the limit of " $f$-feasible" states - states of the economy that are feasible by trade only within coalitions consisting of finite numbers of consumers. The members of a coalition may divide into many (but a finite number of) jurisdictions, each providing public projects for their membership, but feasibility requires that trade of private goods occurs only among members of a coalition. Thus, we must define coalition structures relative to a given jurisdiction structure $\pi$. A coalition structure will be denoted by $\xi$.

Let $S$ be a measurable subset of $N$. A pair $(\xi, \pi)$ is a coalition-jurisdiction structure of $S$ if $\pi$ is a jurisdiction structure and:

1. $\xi$ is a coarsening of $\pi$ that is, for each coalition $W \in \xi$,

$$
W=\cup_{k} \pi^{k}
$$

for some finite collection of jurisdictions $\left\{\pi^{k}\right\}$;

\footnotetext{
${ }^{10}$ Private goods are only endowed; they are not produced. Production of private goods could be incorporated, but this would distract from the issues treated in this paper.

${ }^{11}$ Note that we are taking inputs as non-negative. This is to facilitate an "input accounting device" introduced later.

${ }^{12}$ Note that this does not imply compactness of the set of public projects $\mathcal{X}^{\text {public }}$.
} 
2. $\xi$ is a measurement consistent partition of $S$.

Note that condition 1 . ensures that each coalition $W \in \xi$ consists only of a finite number of consumers.

Let $S$ be a measurable subset of $N$, either finite or infinite, and let $(\xi, \pi)$ be a coalition-jurisdiction structure of $S$. A feasible state for $S$ relative to $(\xi, \pi)$ is a list $((\xi, \pi), X, Y, Z, U)$ where $X: S \rightarrow \mathbb{R}_{+}^{L}$ is a private goods consumption mapping, $Y: S \rightarrow \mathcal{X}^{\text {public }}$ is a public projects consumption mapping, and $Z$ : $S \rightarrow \mathbb{R}^{L}$ is an input accounting device, such that:

1. For almost all $i, j \in S$ if $\pi_{i}=\pi_{j}$ then $Y(i)=Y(j)$ (if two consumers are in the same jurisdiction then they consume the same public projects).

2. For almost all $i \in S,\left(X(i), Y(i)\right.$, pro $\left.\left(\pi_{i}\right)\right) \in \mathcal{X}_{\kappa(i) \tau(i)}$ (except for possibly a set of measure zero, the consumption bundle of each consumer is in his consumption set).

3. The public projects consumption and production mappings are feasible:

(a) For almost all $i \in S$ it holds that $\left(\sum_{j \in \pi_{i}} Z(j), Y(j)\right) \in P\left(\operatorname{pro}\left(\pi_{i}\right)\right)$; and

(b) The distribution of private goods is feasible. That is, for each coalition $W \in \xi$ it holds that

$$
\sum_{i \in W}\left(\omega^{0}(i)-X(i)-Z(i)\right) \geq 0 .
$$

4. $U: S \rightarrow \mathbb{R}$ is a mapping satisfying

$$
U(i)=u_{i}\left(X_{i}, Y_{i}, \operatorname{pro}\left(\pi_{i}\right)\right) \text { for each } i \in N
$$

except possibly for a subset of measure zero.

\section{Remarks.}

(1) Note that in the above definition we assigned to each individual $i$ inputs $Z(i)$ of private goods used to produce public projects in the jurisdiction containing that individual - inputs are indexed by individuals - so to sum inputs, we can sum over individuals. Thus, total input in jurisdiction $\pi_{i}$ of 
private goods into production of public projects is given by $\sum_{j \in \pi_{i}} Z(j)$. This accounting device $Z$ is simply for convenience.

(2) Condition 3. reflects our view that trade takes place between individual consumers or within finite groups of consumers. We take a sum over individuals rather than an integral.

(3) Condition 4. simply introduces some notation that will be convenient and useful.

We will now define feasible states of the economy for a measurable subset $S \subset N$ and a jurisdiction structure $\pi$. Define $F_{S}(\pi)$ by

$$
\begin{gathered}
F_{S}(\pi) \stackrel{\text { def }}{=}\{(X, Y, Z, U) \text { : there is a coalition-jurisdiction structure } \\
(\xi, \pi) \text { and a feasible state }\left((\xi, \pi), X^{\prime}, Y^{\prime}, Z^{\prime}\right) \text { relative to }(\xi, \pi) \\
\text { such that } \left.X=X^{\prime}, Y^{\prime}=Y, Z=Z^{\prime} \text { and } U^{\prime}=U\right\} .
\end{gathered}
$$

The set $F_{S}(\pi)$ includes all feasible states relative to a given jurisdiction structure $\pi$. We now take unions over all jurisdiction structures and limits. Define the sets $F_{S}^{*}(\pi)$, and $F_{S}^{*}$, by

$$
\begin{array}{r}
F_{S}^{*}(\pi) \stackrel{\text { def }}{=}\left\{\left(X^{*}, Y^{*}, Z^{*}, U^{*}\right): \text { for some sequence }\left\{\left(X^{\nu}, Y^{\nu}, Z^{\nu}, U^{\nu}\right)\right\} \text { in } F_{S}(\pi),\right. \\
\left.\left\{\left(X^{\nu}, Y^{\nu}, Z^{\nu}, U^{\nu}\right)\right\} \text { converges in measure to }\left(X^{*}, Y^{*}, Z^{*}, U^{*}\right)\right\}
\end{array}
$$

and

$$
F_{S}^{*} \stackrel{\text { def }}{=} \bigcup_{\pi} F_{S}^{*}(\pi)
$$

The set $F_{S}^{*}{ }^{13}$ consists of the feasible states of the economy for $S$.

Our next assumption is crucial for existence of equilibrium and is an adaptation of an assumption of the same name in earlier research on finite

\footnotetext{
${ }^{13}$ See Hammond, Kaneko and Wooders (1989) for motivation for taking the closure of $F_{S}(\pi)$ with respect to convergence in measure.
} 
economies with one private good and in cooperative games with many consumers. ${ }^{14}$ We assume:

(A.4) Strict small group effectiveness (SSGE): There is a bound $B$ such that for each $\left(X^{*}, Y^{*}, Z^{*}, U^{*}\right) \in F_{S}^{*}$ there is a jurisdiction structure $\pi$ such that $|G| \leq B$ for all $G \in \pi$ and $\left(X^{*}, Y^{*}, Z^{*}, U^{*}\right) \in F_{S}^{*}(\pi)$.

This assumption ensures that, in a continuum economy allowing all possibilities for gains to trade in private goods to be realized, only jurisdictions bounded in size are required to realize all gains to jurisdiction formation. Note that SSGE as defined here does not limit trade in private goods to finite coalitions. Also, arbitrarily large jurisdictions are not ruled out; it is only assumed that anything large jurisdictions can do can also be achieved by a partition of the consumers into jurisdictions bounded in size. We note that a role of strict small group effectiveness is to ensure that the set $F_{S}^{*}$ is closed. ${ }^{15}$

Example 2. Let us first consider a very simple case with two private goods, $x_{1}$ and $x_{2}$, where half the consumers are endowed with one unit of $x_{1}$ and the other half are endowed with one unit of $x_{2}$ and, for completeness, $\mathcal{X}^{\text {public }}=$ $\{0\}$. All consumers have the same crowding types and all consumers have identical preferences given by

$$
u(x, 0, n)=\left\{\begin{array}{l}
x_{1} x_{2}+\sqrt{n}, n \leq 100 \\
x_{1} x_{2}+10 \text { otherwise }
\end{array}\right.
$$

where $n$ is a finite number of people in a jurisdiction. In any sufficiently large finite economy, with a finite set of consumers, the core is not equal to the competitive outcomes since, because the opportunities for trade in

\footnotetext{
${ }^{14}$ This condition grows out of a condition in Wooders (1983), called 'minimum efficient scale,' following an analogous condition in Novshek and Sonnenschein (1982) for production functions. We note that this condition is used to prove results under a much milder condition of boundedness of the supremum of equal treatment payoffs (per capita boundedness). We refer the reader to Kovalenkov and Wooders (2003) for further discussion and references to this condition.

${ }^{15}$ From SSGE it holds that $F_{S}^{*} \subset \bigcup F_{S}^{*}(\pi)$ where the union is taken only over those partitions $\pi$ with $|S| \leq B$ for all $S \in \pi$. There are only a finite number of distinct profiles with norm less than or equal to $B$. This yields the desired conclusion.
} 
private goods increase as the size of the economy increases, the opportunities for improvement by coalitions correspondingly increase. ${ }^{16}$ Yet, from SSGE, gains to jurisdiction formation are exhausted by finite jurisdictions..

Let $S$ be a measurable subset of $N$. A state of the economy $(\pi, X, Y, Z, U) \in$ $F_{S}^{*}$ has the equal treatment property if there is a subset $S^{0}$ of $S$ of full measure $\left(\mu\left(S^{0}\right)=\mu(S)\right)$ such that:

for almost all $i, j \in N$,

$$
\text { if }(\kappa(i), \tau(i))=(\kappa(j), \tau(j)) \text { then } U(i)=U(j) \text {. }
$$

Let $(\pi, X, Y, Z, U) \in F_{N}^{*}$ be a state of the economy $N$. A measurable subset $S \subset N$ of the total population of consumers improves upon $(\pi, X, Y, Z, U)$ with a feasible state of the economy for $S$, say $\left(\pi^{\prime}, X^{\prime}, Y^{\prime}, Z^{\prime}\right) \in$ $F_{S}^{*}$, if for every $i \in S$,

$$
u_{\tau(i)}\left(X^{\prime}(i), Y^{\prime}(i), \operatorname{pro}\left(\pi_{i}^{\prime}\right)\right)>U(i) .
$$

Consistent with our motivation, we require that improving coalitions to be finite.

The $f$-core, or simply the core, of the economy consists of those states of the economy $(\pi, X, Y, Z, U) \in F_{N}^{*}$ with the property that, for some subset of consumers $N^{0} \subset N$ of full measure, there is no finite coalition $S \subset N^{0}$ that can improve upon $(\pi, X, Y, Z, U)$. The equal-treatment core of the economy consists of those states of the economy $(\pi, X, Y, Z, U) \in F_{N}^{*}$ in the core with the equal treatment property.

To prove nonemptiness of the equal treatment core of the economy we rely on a result due to Kaneko and Wooders (1986). Their result, however, is for cooperative games. In Appendix B we define the game generated by an economy and formally define the $f$-core of a game. Informally, the game derived from the economy is constructed by assigning to each finite coalition

\footnotetext{
${ }^{16}$ For comparison, consider replicating an Edgeworth box economy. As the numbers of participants in the economy increases, there are new opportunities for trade within coalitions since coalitions containing different proportions of participants of each type become possible in the replicated economy. It is because of such increases in the opportunities for trade that the core shrinks to the set of price-taking equilibrium outcomes. In our model, in general, possibilities for trade of private goods increase as coalitions become large.
} 
the utility outcomes that coalition can achieve for its members and the $f$ core of a game consists of those functions $h: N \rightarrow \mathbb{R}_{+}$(interpreted as utility space) with the property that no finite coalition can improve upon $h$.

Theorem 1. Nonemptiness of the core of the game generated by the economy. Under assumptions (A.2) and (A.4), the equal-treatment core of the game generated by the economy is nonempty. ${ }^{17}$

Proof of Theorem 1. The proof of this result is a straightforward application of the main result of Kaneko and Wooders (1986). In Appendix B we provide an informal discussion.

An equilibrium price system for crowding type $c$ is a mapping

$$
\psi_{c}: \mathcal{X}^{\text {public }} \times \mathbb{Z}^{C} \rightarrow \mathbb{R}
$$

The value $\psi_{c}(y, \mathcal{C} \operatorname{pro}(S))$ of the mapping $\psi_{c}$ at $(y, \mathcal{C}$ pro $(S))$ is interpreted as the amount of money that a consumer of crowding type $c$ is required to pay to join a jurisdiction with crowding profile $\mathcal{C} \operatorname{pro}(S)$ and consume the vector $y$ of public projects. A price system $\psi$ for public projects is a collection of price systems, one for each crowding type.

In the following definition, note that, as in the definition of a feasible state, for the purposes of adding up the total input of private goods into public project production, we distribute the private good inputs in a jurisdiction among the members of the jurisdiction.

An equilibrium is a state of the economy $(\pi, X, Y, Z, U) \in F_{N}^{*}$ for $N$, a price system $p \in \mathbb{R}_{+}^{L}$ for private goods, and a price system $\psi$ for public projects such that:

1. For almost all $i \in N,\left(X(i), Y(i), \mathcal{C} p r o\left(\pi_{i}\right)\right) \in \mathcal{X}_{\kappa(i) \tau(i)}$ and

$$
\left.p \cdot X(i)+\psi_{\kappa(i)}\left(Y(i), \mathcal{C} \operatorname{pro}\left(\pi_{i}\right)\right)\right)=p \cdot \omega^{0}(i) .
$$

2. For almost all $i \in N$, for all jurisdictions $G \in \mathcal{F}$ such that $i \in G$, for all possible bundles of private goods $x \in \mathbb{R}_{+}^{L}$ and public projects $y \in$ $\mathcal{X}^{\text {public }}$, if

$$
u_{\tau(i)}(x, y, \operatorname{pro}(G))>U(i)
$$

\footnotetext{
${ }^{17}$ We note that the anonymity assumptions (A.1) and (A.3) are not required for this result.
} 
then

$$
\left.p \cdot x+\psi_{\kappa(i)}(y, \mathcal{C} \operatorname{pro}(G))\right)>p \cdot \omega^{0}(i) .
$$

3. For almost all $i \in N$

$$
\sum_{j \in \pi_{i}} \psi_{\kappa(j)}\left(Y(j), \mathcal{C} \operatorname{pro}\left(\pi_{i}\right)\right)-p \cdot \sum_{j \in \pi_{i}} Z(j)=0
$$

4. For every $G \in \mathcal{F}$, there does not exist $(z, y) \in P(\operatorname{pro}(G))$ such that

$$
\sum_{j \in G} \psi_{\kappa(j)}(y, \mathcal{C} \operatorname{pro}(G))-p \cdot z>0 .
$$

Theorem 2. An equilibrium state of the economy is in the core. If a feasible state of the economy $(\pi, X, Y, Z, U) \in F_{N}^{*}$ and price systems $p$ and $\psi$ constitute an equilibrium, then $(\pi, X, Y, Z, U)$ is in the core.

Proof. See the Appendix. ${ }^{18}$

Next we demonstrate an equal treatment theorem, extending the equaltreatment property of the core of replicated exchange economies and games with strictly effective small groups to continuum economies with local public projects. This depends on our assumption that consumers of the same taste type have the same endowment ${ }^{19}$ and on an additional assumption.

(A.5) Desirability of the endowment of private goods. For each consumer $i$, it holds that:

$$
u_{\tau(i)}\left(\omega^{0}(i), \underline{0}, \operatorname{pro}(\{i\})\right)>u_{\tau(i)}(0, y, \operatorname{pro}(G))
$$

for any $y \in \mathcal{X}^{\text {public }}$ and any jurisdiction $G$ containing consumer $i$.

\footnotetext{
${ }^{18}$ Theorem 2 is proven using the game-theoretic notion of the core, where all members of an improving coalition must be better off. In general, this leads to a larger core than the notion frequently used in economics, where all members of an improving coalition must be at least as well off and one must be strictly better off. The Theorem is also easily proven for this alternative notion of the core.

${ }^{19}$ The equal treatment property of the core has a long history in economics, going back to Shubik (1959) and Debreu and Scarf (1963). The equal-treatment property of the core for replicated NTU games with strictly effective small groups is shown in Wooders (1983, Theorem 3).
} 
Assumption (A.5) dictates that the endowment is preferred to any bundle containing zero private goods. ${ }^{20}$ This assumption ensures that in an individually rational state of the economy, each individual will consume some positive amount of private goods.

Theorem 3. The equal treatment property of the core. Assume (A.2), (A.4) and (A.5). Then there exists a feasible state of the economy $(\pi, X, Y, Z, U)$ in the core. Moreover, there is a subset $N^{0} \subset N, \mu\left(N^{0}\right)=\mu(N)$, such that for every pair of consumers $i, j \in N^{0}$ satisfying $\tau(i)=\tau(j)$ and $\kappa(i)=\kappa(j)$ it holds that

$$
U(i)=U(j) .
$$

Proof of Theorem 3. See the Appendix.

Theorem 3 is used in our proof of the equivalence of the core and the equilibrium states of the economy.

Theorem 4. Equivalence of the equal-treatment core and the equilibrium states of the economy. Let $(\pi, X, Y, Z, U) \in F_{N}^{*}$ be an equal-treatment core state of the economy satisfying (A.1)-(A.5). Then there is a price system $p$ for private goods and a price system $\psi$ for public projects such that $(\pi, X, Y, Z, U), p$ and $\psi$ constitute an equilibrium.

Proof of Theorem 4. See the Appendix.

Theorem 5. Existence of equilibrium. Assume that the economy satisfies (A.1)-(A.5). Then there exists an equilibrium for the economy.

Proof of Theorem 5. From Theorem 3 the equal-treatment core is nonempty. From Theorem 4 every state of the economy in the equal-treatment core is an equilibrium state. Thus, an equilibrium exists.

The following Theorem concludes our results.

Theorem 6. Core-equilibrium equivalence. Assume that the economy satisfies (A.1)-(A.5). Then an equilibrium exists and the set of equilibrium states of the economy is equivalent to the core.

\footnotetext{
${ }^{20}$ This assumption also appears in Hammond, Kaneko and Wooders (1989), Kaneko and Wooders (1989) and Ellickson et al. (1999).
} 
Proof of Theorem 6. This is immediate from Theorem 2, Theorem 3, and Theorem 4.

\section{Some further remarks on the literature}

Before concluding, we contrast our work to some other approaches to continuum economies with small effective groups.

1. An especially interesting aspect of our work is that equilibrium jurisdictions may be large, that is, for any positive integer $n$ in an equilibrium state of the economy there may exist a jurisdiction with more than $n$ members. Strict small group effectiveness only ensures that all potential gains to jurisdiction formation can be realized by states of the economy with jurisdictions no larger than some fixed, finite bound; it does not rule out larger jurisdictions.

2. Our notion of feasibility follows Kaneko and Wooders (1986) and Hammond, Kaneko and Wooders (1989). It is well-known that in models with a continuum of consumers and finite, but unbounded coalition sizes, the set of feasible allocations may not be closed (cf. Hammond, Kaneko and Wooders 1989). Thus, the feasible set is taken as the closure of the set of allocations (or, for games, the set of payoffs) that are achievable by trade only within finite coalitions. When this closure is taken, the set of allocations that are " $f$-feasible" is equivalent to the set of Aumann-feasible allocations, as in Aumann (1964) (see Kaneko and Wooders 1986). For the purposes of the current paper, we wish to treat finite jurisdictions but to allow the same set of feasible trades as in the extant literature on economies with private goods. Thus, relative to any jurisdiction structure $\pi$ we allow trade within arbitrarily large coalitions (coarsening of the jurisdiction structure) and then take the closure with respect to convergence in measure. Relative to that jurisdiction structure, this allows us to capture the same set of feasible trades as in the Aumann approach to the continuum. We then assume that all gains to forming jurisdictions are realizable by jurisdictions structures bounded in size. This allows all possible gains to trade of private goods to be captured by arbitrarily large coalitions, while maintaining the feature that jurisdictions be finite. 
3. An important feature of our research is that jurisdiction sizes are unbounded. Thus, for a given composition of the jurisdiction, there may be constant returns to increasing size of the jurisdiction. This is an important aspect of our research, creating new problems for existence of equilibrium and requiring some subtlety and new approaches in our proof techniques. In particular, even though we can ignore sets of consumers of measure zero and thus effectively have 'thickness' of the total consumer set, the equal treatment property of all outcomes in the core - essential for equivalence of the set of outcomes in the core and the set of equilibrium outcomes - is not immediate. Moreover, the percentages of consumers of each type could be bounded away from zero and the same difficulties would appear. This is in contrast, for example, to the situation of games with transferable utility and what motivates our particular form of strict small group effectiveness.

We note that another recent paper, Allouch and Wooders (2008), al-

lows unbounded jurisdiction sizes in large finite economies. There are a number of distinctions between their work and ours; Allouch and Wooders treat large finite economies and the core notion introduced involves communication costs in the formation of jurisdictions. Also, they treat economies where consumers may belong to multiple clubs or jurisdictions. A major difference between the two models is that Allouch and Wooders allow forever strictly increasing returns to jurisdiction size and the only optimal jurisdiction structure may be the jurisdiction consisting of the entire population. This creates difficulties in the definition of a limit economy. Moreover, with forever increasing returns to jurisdiction size, exact equal treatment of identical consumers need not hold and equilibrium need be only approximately Pareto efficient.

4. There is an important difference between the approach of this paper, allowing public projects with minimal assumptions on production, and the approach of Wooders $(1985,1997)$ for growing sequences of finite economies. $^{21}$ Recall that Wooders' model required that production sets for public goods be closed convex cones and that pricing was dif-

\footnotetext{
${ }^{21}$ Wooders $(1985,1997)$ use the same proof of convergence, except the later paper shows that the prices for public goods derived in the proofs of the earlier papers are Lindahl and also determine admission prices. Conley and Wooders (1997) discusses the differences between Lindahl pricing and admission pricing.
} 
ferentiated - that is, prices for public goods were based on consumers' types where "type" included taste type. Our model does not require these restrictions. In the course of our proof, following Wooders's earlier papers, we define preferred sets of net trades of private goods for jurisdictions, Wooders obtained existence of equilibrium prices for private goods by separating the preferred sets of jurisdictions from the origin. We also use such a separating hyperplane argument, but we separate only preferred sets for jurisdictions in the core from the origin. From the prices for private goods thus determined, we are able to construct prices for public projects for all jurisdictions. To the best of our knowledge, there is no precedent for this technique.

5. Our notion of feasibility follows Kaneko and Wooders (1986) and Hammond, Kaneko and Wooders (1989). It is well-known that in models with a continuum of consumers and finite, but unbounded coalition sizes, the set of feasible allocations may not be closed (cf. Hammond, Kaneko and Wooders 1989). Thus, the feasible set is taken as the closure of the set of allocations (or, for games, the set of payoffs) that are achievable by trade only within finite coalitions. When this closure is taken, the set of allocations that are " $f$-feasible" is equivalent to the set of Aumann-feasible allocations, as in Aumann (1964) (see Kaneko and Wooders 1986). For the purposes of the current paper, we wish to treat finite jurisdictions but to allow the same set of feasible trades as in the extant literature on economies with private goods. Thus, relative to any jurisdiction structure $\pi$ we allow trade within arbitrarily large coalitions (coarsening of the jurisdiction structure) and then take the closure with respect to convergence in measure. Relative to that jurisdiction structure, this allows us to capture the same set of feasible trades as in the Aumann approach to the continuum. We then assume that all gains to forming jurisdictions are realizable by jurisdictions structures bounded in size. This allows all possible gains to trade of private goods to be captured by arbitrarily large coalitions, while maintaining the feature that jurisdictions be finite.

6. An important feature of our research is that jurisdiction sizes are unbounded. Thus, for a given composition of the jurisdiction, there may be constant returns to increasing size of the jurisdiction. This is an important aspect of our research, creating new problems for existence 
of equilibrium and requiring some subtlety and new approaches in our proof techniques. In particular, even though we can ignore sets of consumers of measure zero and thus effectively have 'thickness' of the total consumer set, the equal treatment property of all outcomes in the core - essential for equivalence of the set of outcomes in the core and the set of equilibrium outcomes - is not immediate. Moreover, the percentages of consumers of each type could be bounded away from zero and the same difficulties would appear. This is in contrast, for example, to the situation of games with transferable utility and what motivates our particular form of strict small group effectiveness.

We note that another recent paper, Allouch and Wooders (2008), allows unbounded jurisdiction sizes in large finite economies. There are a number of distinctions between their work and ours; Allouch and Wooders treat large finite economies and the core notion introduced involves communication costs in the formation of jurisdictions. Also, they treat economies where consumers may belong to multiple clubs or jurisdictions. A major difference between the two models is that Allouch and Wooders allow forever strictly increasing returns to jurisdiction size and the only optimal jurisdiction structure may the jurisdiction consisting of the entire population. This creates difficulties in the definition of a limit economy. Moreover, with forever increasing returns to jurisdiction size, exact equal treatment of identical consumers need not hold and equilibrium need be only approximately Pareto efficient.

7. We conclude by noting the difference between the $f$-core and the "finite core" (see Keiding 1976 and references therein). The finite core allows improvement by finite coalitions, but imposes a feasibility requirement independent of any measure on the set of consumers. Thus, relative scarcities of consumer types and commodities - the sine qua non of economics - are ignored. This has the consequence that the finite core is not necessarily the limit of approximate cores of large economies. ${ }^{22}$ Consider, for example, a sequence of finite matching games with transferable utility and with two types of consumers - males and females. Suppose there are twice as many males as females. Since females are

\footnotetext{
${ }^{22}$ Kaneko and Wooders (1989) show that for private goods economies the continuum with finite coalitions is the limit of large finite economies with relatively small effective coalitions. This also holds for games.
} 
relatively scarce, for any finite game the core assigns all gains to marriage to females; males receive only their individually rational payoff. Now suppose there is a continuum of consumers and twice as many males as females. The $f$-core assigns all gains to marriage to females. In contrast, with Keiding's notion of feasibility, any Pareto-optimal and individually rational equal-treatment payoff is in the finite core.

\section{Conclusions}

The point of this paper has been to provide a model allowing confirmation of Tiebout's hypothesis that when public goods are local, markets are able to decentralize the efficient outcomes. Traditionally, there has been a trade-off in the literature. On the one hand, it is has been widely demonstrated that, without special assumptions, both the core and competitive equilibrium may fail to exist in finite economies. Defining equilibrium notions that can be shown to exist, on the other hand, typically involves restricting consumers' alternatives to a subset of all feasible allocations (for example, requiring that consumers always share the cost of public goods equally or restricting the menu of admissible clubs to an arbitrary, finite subset of the feasible set). Thus, although these equilibrium exist, at best they are only to be Pareto optimal within the constraints imposed and not over the whole feasible set. In addition, it is often the case that many other equilibria also exist which do not even satisfy this constrained optimality.

The intuition for our results comes from the Tiebout literature dealing with large finite economies. This literature suggests that the failure of existence of equilibrium is caused by the presence of a group of "left-over" consumers who cannot find placement in optimal jurisdictions. In the continuum limit, the problem of left-over consumers disappears. Extending the intuition of large finite economies to economies with a continuum of consumers creates both technical and intuitive problems. Much of the literature supposes that, in equilibrium, consumers end up in a finite number of infinitely large jurisdictions. This reflects neither everyday observation, nor is it the limiting case of the $\varepsilon$-equilibrium.

These considerations motivate our use of an $f$-core approach in an environment with local public goods and production of both public and private goods. This economy allows the multiple private goods to be traded freely across jurisdictional boundaries, but requires that crowding and public 
projects be consumed only within jurisdictions. The space of public projects we consider is quite abstract and does not require any linear structure, but includes standard infinitely divisible public goods as a special case. The most innovative part of this paper is showing the equivalence of the equaltreatment core and the competitive outcomes, thereby obtaining existence of equilibrium in a novel way.

Our main result is that under fairly standard conditions on production and preferences, the core is nonempty and is equivalent to the set of anonymous admission price equilibrium outcomes. Thus, Tiebout's hypothesis is confirmed in the sense that except for at most a negligible fraction of consumers, competitive equilibria exist and are first best.

There are several ways in which the research of this paper might be furthered. In particular, what prevents the proving a second welfare theorem in the case of a finite Tiebout economy is the general failure of existence of competitive equilibrium. Thus, we speculate that it should be possible to prove a second welfare theorem in the generality of our model. We have also treated crowding characteristics as exogenously given (for example gender, race or intelligence might be externality producing characteristics that are exogenous to consumers). It would be interesting to extend this model to endogenously chosen externality producing characteristics like skills or being a smoker as in Conley and Wooders (1997), for example. Finally, it should be possible to prove results similar to those given in this paper when consumers are allowed to join more than one club at a time. The modeling challenge, as we perceive it, is to maintain measurement-consistency while allowing consumers to join an arbitrary number of clubs, each of which may be able to produce an arbitrary level of public goods.

\section{Appendix A}

For the convenience of the reader, we first list the main (numbered) assumptions:

(A.1) Taste anonymity in consumption (TAC): For all $x \in \mathbb{R}_{+}^{L}, y \in \mathcal{X}^{\text {public }}$ and all $G, G^{\prime} \in \mathcal{F}$ such that $(x, y, \operatorname{pro}(G)),\left(x, y, \operatorname{pro}\left(G^{\prime}\right)\right) \in \mathcal{X}_{c t}$ and $\mathcal{C}$ pro $(G)=\mathcal{C}$ pro $\left(G^{\prime}\right)$ it holds that $u_{t}(x, y, \operatorname{pro}(G))=u_{t}\left(x, y, \operatorname{pro}\left(G^{\prime}\right)\right)$.

(A.2) Given a jurisdiction $G \in \mathcal{F}$ and a vector $\bar{z} \in \mathbb{R}_{+}^{L}$ the set $\{(z, y) \in$ $\left.P(\operatorname{pro}(G)): z \in R_{+}^{L}, z \leq \bar{z}\right\}$ is compact. 
(A.3) Taste anonymity in production (TAP): For all $G, G^{\prime} \in \mathcal{F}$ such that $\mathcal{C}$ pro $(G)=\mathcal{C}$ pro $\left(G^{\prime}\right)$ it holds that $P(\operatorname{pro}(G))=P\left(\operatorname{pro}\left(G^{\prime}\right)\right)$.

(A.4) Small groups are strictly effective (SSGE), that is, there is a bound $B$ such that for each $\left(X^{*}, Y^{*}, Z^{*}, U^{*}\right) \in F_{S}^{*}$ there is a jurisdiction structure $\pi$ such that $|G| \leq B$ for all $G \in \pi$ and $\left(X^{*}, Y^{*}, Z^{*}, U^{*}\right) \in F_{S}^{*}(\pi)$. This assumption ensures that, in a continuum economy, where all possibilities for gains to trade in private goods can be realized, only jurisdictions bounded in size are required to realize all gains to jurisdiction formation.

(A.5) Desirability of the endowment of private goods. For each consumer $i$, it holds that:

$$
u_{\tau(i)}\left(\omega^{0}(i), \underline{0}, \operatorname{pro}(\{i\})\right)>u_{\tau(i)}(0, y, \operatorname{pro}(G))
$$

for any $y \in \mathcal{X}^{\text {public }}$ and any jurisdiction $G$ containing consumer $i$.

Theorem 2. An equilibrium state of the economy is in the $f$-core. If a feasible state of the economy $(\pi, X, Y, Z, U)$ and price systems $p$ and $\psi$ constitute an equilibrium, then $(\pi, X, Y, Z, U)$ is in the $f$-core of the economy.

Proof of Theorem 2. Suppose that a feasible state of the economy $(\pi, X, Y, Z, U)$, a price system $p \in \mathbb{R}^{L}$ for private goods and a price system $\psi$ for public projects constitute an equilibrium. Thus, there exists a subset $N^{0}$ of $N$ with the property that $\mu\left(N^{0}\right)=\mu(N)$ and, for all jurisdictions $\pi_{i}, i \in N^{0}$, conditions 1-4 of the definition of an equilibrium are satisfied. Suppose the equilibrium is not in the core. Then there is at least one finite coalition, say $W \subset N^{0}$, a feasible state of the economy for $W$, say $\left(\pi^{\prime}, X^{\prime}, Y^{\prime}, Z^{\prime}\right)$, that can improve upon $(\pi, X, Y, Z, U)$ for its members. That is, for every consumer $i \in W$ it holds that

$$
\begin{gathered}
u_{\tau(i)}\left(X^{\prime}(i), Y^{\prime}(i), \operatorname{pro}\left(\pi_{i}^{\prime}\right)\right)>u_{\tau(i)}\left(X(i), Y(i), \operatorname{pro}\left(\pi_{i}\right)\right) \text { and } \\
\sum_{i \in W}\left(\omega^{0}(i)-X(i)-Z(i)\right)=0 .
\end{gathered}
$$

Therefore, for every $i \in W$ it holds that 


$$
p \cdot X^{\prime}(i)+\psi_{\kappa(j)}\left(Y^{\prime}(i), \mathcal{C} \operatorname{pro}\left(\pi_{i}^{\prime}\right)\right)>p \cdot \omega^{0}(i) .
$$

From the condition of equilibrium that profits are nonpositive for jurisdictions in $\pi^{\prime}$, it holds for every $i \in W$ that

$$
\sum_{j \in \pi_{i}^{\prime}} \psi_{\kappa(j)}\left(Y^{\prime}(j), \mathcal{C} \operatorname{pro}(G)\right)-p \cdot \sum_{j \in \pi_{i}^{\prime}} Z^{\prime}(j) \leq 0
$$

From the above expressions it now follows that

$$
\begin{aligned}
& p \cdot \sum_{i \in W} \omega^{0}(i)<p \cdot \sum_{i \in W} X^{\prime}(i)+\sum_{i \in W} \psi_{\kappa(i)}\left(Y^{\prime}(i), \mathcal{C} \operatorname{pro}\left(\pi_{i}^{\prime}\right)\right) \\
& \leq p \cdot \sum_{i \in W} X^{\prime}(i)+p \cdot \sum_{i \in W} Z^{\prime}(i) .
\end{aligned}
$$

But, from feasibility, $W$ it holds that

$$
p \cdot \sum_{i \in W} \omega^{0}(i) \geq p \cdot \sum_{i \in W} X^{\prime}(i)+p \cdot \sum_{i \in W} Z^{\prime}(i),
$$

and therefore

$$
p \cdot \sum_{i \in W} \omega^{0}(i) \geq p \cdot \sum_{i \in W} X^{\prime}(i)+p \cdot \sum_{i \in W} Z^{\prime}(i)>p \cdot \sum_{i \in W} \omega^{0}(i)
$$

the desired contradiction.

Theorem 3. The equal treatment property of the core. Assume (A.2), (A.4) and (A.5). Then there exists a feasible state of the economy $(\pi, X, Y, Z, U)$ in the core of the economy. Moreover, there is a subset $N^{0} \subset N, \mu\left(N^{0}\right)=$ $\mu(N)$, such that for every pair of consumers $i, j \in N^{0}$ satisfying $\tau(i)=\tau(j)$ and $\kappa(i)=\kappa(j)$ it holds that

$$
u_{\tau(i)}\left(X(i), Y(i), \operatorname{pro}\left(\pi_{i}\right)\right)=u_{\tau(j)}\left(X(j), Y(j), \operatorname{pro}\left(\pi_{j}\right)\right) .
$$

\section{Proof of Theorem 3.}


Let $h: N \rightarrow \mathbb{R}$ be in the equal treatment core of the game generated by the economy. (See Theorem 1 for complete definitions.) Note that by Theorem 1, proven in Appendix B, such a function exists. Then there is a subset of full measure, say $N^{\prime}\left(\mu\left(N^{\prime}\right)=\mu(N)\right)$, and a sequence of functions $\left\{h^{\nu}\right\}$ converging in measure to $h$ such that there exists a sequence of coalition-jurisdiction structures $\left\{\left(\xi^{\nu}, \pi^{\nu}\right)\right\}_{\nu}$ and, for each $\nu$, a feasible state $\left(\left(\xi^{\nu}, \pi^{\nu}\right), X^{\nu}, Y^{v}, Z^{\nu}\right)$ relative to $\left(\xi^{\nu}, \pi^{\nu}\right)$, satisfying

$$
u_{\tau(i)}\left(X^{\nu}(i), Y^{\nu}(i), \operatorname{pro}\left(\pi_{i}^{\nu}\right)\right) \geq h^{\nu}(i) \text { for each } i \in N^{\prime} .
$$

Let $B$ be the bound given in the definition of SSGE. Let $\left\{p r o^{1}, \ldots\right.$, pro $^{k}, \ldots$, pro $\left.^{K}\right\}$ denote the set of all profiles satisfying pro $^{k} \in \mathbb{Z}^{C T}$ and $\|$ pro $o^{k} \| \leq B$, where $\|$ pro $^{k} \|:=\sum_{c t}$ pro $_{c t}^{k}$. We consider the space $A=\mathbb{R}^{C T K}$ where, as previously defined, $C$ is the number of crowding types, $T$ is the number of taste types and $K$ is the number of profiles bounded in size by $B$. Let $a=$ $\left(a^{1}, \ldots, a^{k}, \ldots, a^{K}\right)$ be a vector where, for each $k, a^{k}=\left(a_{1,1}^{k}, \ldots, a_{c, t}^{k}, \ldots, a_{C, T}^{k}\right)$ and for each $(c, t)$ pair, $a_{c, t}^{k}=$ pro $_{c, t}^{k} \in \mathbb{Z}$. For each consumer $i$, for each $\nu$, let $a^{\nu}(i) \in A$ be a vector with components $a_{c, t}^{k}=1$ if $\kappa(i)=c ; \tau(i)=t$ and $\operatorname{pro}\left(\pi_{i}^{\nu}\right)=\operatorname{pro}^{k}$ and $a_{c, t}^{k}=0$ otherwise. Also, let $\overline{1} \in A$ be such that $a_{c, t}^{k}$ equals one for all $c, t$ and $k$.

Now, we consider the following sequence $\left(X_{i}^{\nu}, Y_{i}^{\nu}, Z_{i}^{\nu}, a_{i}^{\nu}\right)$. From feasibility we have

$$
\int\left(X^{\nu}(i)+Z^{\nu}(i), a^{\nu}(i)\right) d \mu \leq \int(w(i), \overline{1}) d \mu .
$$

We can assume without loss of generality that $\int_{N}\left(X^{\nu}(i)+Z^{\nu}(i), a^{\nu}(i)\right) d \mu$ converges. We can then apply Fatou's Lemma in $m$-dimensions (Hildenbrand, 1974 , p. 69, Lemma 3 ) to this sequence and conclude that there is an integrable function $(\bar{X}+\bar{Z}, \bar{a})$ and a set $N^{0}$ of full measure such that $(\bar{X}(i)+$ $\bar{Z}(i), \bar{a}(i)) \in \operatorname{LimSup}\left(X^{\nu}(i)+Z^{\nu}(i), a^{\nu}(i)\right)$ for each $i \in N^{0}$, and

$$
\int(\bar{X}(i)+\bar{Z}(i)) d \mu \leq \lim \int\left(X^{\nu}(i)+Z^{\nu}(i)\right) d \mu \text { and } \int \bar{a}(i) d \mu=\lim \int a^{\nu}(i) d \mu
$$

Since $a^{\nu}(\cdot)$ has a finite range, any convergent subsequence of $\left\{a^{\nu}\right\}$ is constant after some rank; therefore $\left(X^{\nu}(i), Y^{\nu}(i), Z^{\nu}(i)\right) \in F_{N}^{*}(\pi)$ for some jurisdiction structure $\pi$. I follows from strict small group effectiveness that $(\bar{X}(i), \bar{Y}(i), \bar{Z}(i)) \in F_{N}^{*}$. Moreover, from Fatou's Lemma there is a subsequence $\left(X^{\nu \lambda}(i), Y^{\nu \lambda}(i), Z^{\nu \lambda}(i)\right)$ such that

$$
u_{\tau(i)}\left(X^{\nu \lambda}(i), Y^{\nu \lambda}(i), \operatorname{pro}\left(\pi_{i}\right)\right) \geq u_{\tau(i)}^{\nu \lambda} .
$$


Taking the limit we obtain

$$
u_{\tau(i)}\left(\bar{X}(i), \bar{Y}(i), \operatorname{pro}\left(\pi_{i}\right)\right) \geq u_{\tau(i)} .
$$

We conclude that $(\pi, X, Y, Z, U)$ belongs to the $f$-core of the economy.

Next we will show the equal treatment property for some allocations in the core. From the definition of the $f$-core of a game we know that the core payoff for each consumer $i$ is individually rational, that is to say,

$$
h(i) \geq u_{\tau(i)}\left(\omega^{0}(i), \underline{0}, \operatorname{pro}(\{i\})\right) .
$$

From (A.5) (Desirability of the endowment of divisible private goods) it follows that:

$$
u_{\tau(i)}\left(\omega^{0}(i), \underline{0}, \operatorname{pro}(\{i\})\right)>u_{\tau(i)}\left(0, \bar{Y}(i), \operatorname{pro}\left(\pi_{i}\right)\right) .
$$

Therefore one obtains for each consumer $i$

$$
u_{\tau(i)}\left(\bar{X}(i), \bar{Y}(i), \operatorname{pro}\left(\pi_{i}\right)\right) \geq h(i)>u_{\tau(i)}\left(0, \bar{Y}(i), \operatorname{pro}\left(\pi_{i}\right)\right) .
$$

From the continuity of the utility functions for each consumer $i$ there exists $0<\lambda_{i} \leq 1$ such that

$$
u_{\tau(i)}\left(\lambda_{i} \bar{X}(i), \bar{Y}(i), \operatorname{pro}\left(\pi_{i}\right)\right)=h(i)
$$

We set $\bar{X}^{\prime}(i)=\lambda_{i} \bar{X}(i)$. It is clear that $\left(\pi, \bar{X}^{\prime}, \bar{Y}, \bar{Z}, U\right)$ belongs to the $f$-core and satisfies the equal treatment property.

Theorem 4. Equivalence of the equal-treatment core and the equilibrium states of the economy. Let $(\pi, X, Y, Z, U)$ be an equal-treatment core state of the economy satisfying (A.1)-(A.5). Then there is a price system $p$ for private goods and a price system $\psi$ for public projects such that $(\pi, X, Y, Z, U), p$ and $\psi$ constitute an equilibrium.

Sketch of the proof of Theorem 4. In Step 1, we obtain the existence of a price system, say $p$, for private goods for a finite approximating economy. To obtain this result, we follow a technique arising from Debreu-Scarf (1963), of 
separating preferred sets from the origin. Instead of considering the preferred sets of individual consumers as in Debreu-Scarf (1963) and Foley (1970), we consider preferred sets for jurisdictions, as in Wooders $(1985,1997)$ and treat the preferred sets of private goods for jurisdictions, for all jurisdictions in $\pi$. A point in the preferred set of a jurisdiction is an amount of private goods sufficiently large so that, with this amount of resources, it is possible for all members of the jurisdiction to be better off than they are in the state of the economy in the core. In Step 2, it is verified that $p$ satisfies the conditions of the Theorem. Finally, in Step 3, using the same techniques as in Conley and Wooders (1997) for a one-private-good case, the price system $\psi$ is constructed from the price system $p$. Step 4 completes the proof by showing that all the properties of a competitive equilibrium are satisfied.

\section{Proof of Theorem 4.}

Preliminaries. Let $(\pi, X, Y, Z, U)$ be an equal-treatment state of the economy in the core. From the definition of the equal treatment property, $(\pi, X, Y, Z, U)$ assigns almost all consumers of the same type the same utility levels. For a consumer of type $(c, t)$ let $\bar{U}(c, t)$ denote this utility level.

Let $N^{0}$ be a subset of $N$ with the property that $\mu\left(N^{0}\right)=\mu(N)$ and, if $i \in N^{0}$ and $(\kappa(i), \tau(i))=(c, t)$, then there is an infinite number of consumers in $N^{0}$ of type $(c, t)$.

\section{Step 1.}

Let $\mathcal{G}$ denote the collection of all possible jurisdictions $G$ contained in $N^{0}$. For each $G \in \mathcal{G}$ let $G$ denote the set of private goods bundles $b$ in $\mathbb{R}^{L}$ with the properties that, for each $i \in G$, there is an $x^{i} \in \mathbb{R}_{+}^{L}$ such that:

$$
\begin{gathered}
u_{\tau(i)}(X(i), Y(i), \operatorname{pro}(G))>\bar{U}(c, t) \text { and } \\
b=\sum_{i \in G}\left(X(i)-Z(i)-w^{0}(i)\right),
\end{gathered}
$$

where $\bar{U}(c, t)$ is the utility assigned to consumers of type $(c, t)$ in the core allocation. The set $G$ is a subset of $\mathbb{R}^{L}$, called the preferred set for $G$. For jurisdiction $G,{ }_{G}$ is the set of aggregate net trades (or transfers) of private goods with the property that there is some production of public projects and some distribution of private goods so that, for each member of $G$, the given 
allocation is preferred to the allocation which he is assigned in the state of the economy $(\pi, X, Y, Z, U)$. (Note that $G$ may be empty.)

Let $\subset \mathbb{R}^{L}$ denote the convex hull of the union $\cup_{G \in \mathcal{G}} \quad G$. Arguing by contradiction, we next show that $0 \notin$. So let us suppose that $0 \in$. Then, since $\subset \mathbb{R}^{L}$ is the convex hull of the sets $\{G\}$ :

(i) there is a finite collection of jurisdictions $\mathcal{G}^{\prime} \subset \mathcal{G}$ and a convex combination of weights $\lambda_{G}, G \in \mathcal{G}^{\prime}$ satisfying $0<\lambda_{G} \leq 1$ and $\sum_{G \in \mathcal{G}^{\prime}} \lambda_{G}=1$.

(ii) for each $G \in \mathcal{G}^{\prime}$ and each $i \in G$, there is a private goods consumption bundle $x^{i}$ such that:

(a) $0=\sum_{G \in \mathcal{G}^{\prime}}\left[\lambda_{G}\left(\sum_{i \in G}\left(x^{i}-Z(i)-\omega^{0}(i)\right)\right)\right]$, and

(c) $u_{\tau(i)}\left(x^{i}, Y(i), \operatorname{pro}(G)\right)>\bar{U}(c, t)$.

From continuity and monotonicity of preferences, the sets $G$ are open. Therefore, if any of the weights $\lambda_{G}$ are irrational, we can perturb the allocations of private goods $x^{i}$ so that (a) and (b) are satisfied with rational weights $\lambda_{G}$. Thus, we suppose, without loss of generality, that the weights $\lambda_{G}, G \in \mathcal{G}^{\prime}$, are all rational numbers.

Let $r$ be an integer such that $r \lambda_{G}$ is an integer for all $G \in \mathcal{G}^{\prime}$. It holds that $r \sum_{G \in \mathcal{G}^{\prime}} \lambda_{G} \operatorname{pro}(G) \in \mathbb{Z}^{C T}$. Let $W$ denote a finite set of consumers of consumers with the same number of consumers of each type $(c, t)$ as $r \sum_{G \in \mathcal{G}^{\prime}} \lambda_{G} \operatorname{pro}(G)$, that is

$$
\operatorname{pro}(W)=r \sum_{G \in \mathcal{G}^{\prime}} \lambda_{G} \operatorname{pro}_{c t}(G) .
$$

Therefore the coalition $W$ can improve upon the state of the economy $(\pi, X, Y, Z, U)$ for its members and we have a contradiction to the supposition that $(\pi, X, Y, Z, U)$ is in the core. Thus, $0 \notin$.

From the fact that is convex and $0 \notin$ it follows that there exists a price system $p$ for private goods that separates the preferred sets for jurisdictions $G$ in $\mathcal{G}^{\prime}$ from their affordable net trades of private commodities. From monotonicity of preferences, it follows that $p_{\ell}>0$ for each private good $\ell=1, \ldots, L$.

Step 2. A private-goods price system for the continuum economy.

Let $\pi^{0}$ denote the jurisdiction structure $\pi$ restricted to consumers contained in $N^{0}$. We now show that $p$ satisfies the properties that (a) for almost 
all consumers $i \in N^{0}$ it holds that the jurisdiction $\pi_{i}^{0}$ can afford its bundle of private goods and (b) except possibly for a set of measure zero, no jurisdiction $G \in \pi^{0}$ of consumers could be better off given prices $p$. Observe that for each $G \in \pi^{0}$ from the definition of and the continuity of utility functions it holds that for any $i \in G, \sum_{j \in \pi_{i}^{0}}\left[X(j)+Z(j)-\sum_{j \in \pi_{i}^{0}} \omega^{0}(j)\right]$ is in the closure of $G$. Thus, it follows from the separating hyperplane property of $p$ that

$$
p \cdot \sum_{j \in \pi_{i}^{0}}(X(j)+Z(j)) \geq p \cdot \sum_{j \in \pi_{i}^{0}} \omega^{0}(j)
$$

From feasibility we obtain

$$
\int(X(i)+Z(i)) d \mu \leq \int \omega^{0}(i) d \mu .
$$

Thus, for almost all consumers $i \in N^{0}$,

$$
p \cdot \sum_{j \in \pi_{i}^{0}}(X(j)+Z(j))=p \cdot \sum_{j \in \pi_{i}^{0}} \omega^{0}(j) .
$$

Thus, for almost all consumers $i \in N^{0}$, the jurisdiction $\pi_{i}$ can afford the allocation for its members given by $(\pi, X, Y, Z, U)$.

Step 3. A public-projects price system. We must now construct prices for public projects. Here we follow the techniques of Conley and Wooders (1997). Although that paper only has one private good, once prices for private goods are given the problem becomes quite similar to the problem in the one-private-good case. The following Lemma, demonstrating that any two consumers of the same crowding type in the same jurisdiction must make the same contribution (in terms of monetary worth) to public project provision, is the analogue of Conley and Wooders (1997, Theorem 2).

Lemma 1. Let $(\pi, X, Y, Z, U)$ be a state of the economy in the core. Let $p$ be as determined above. Then for any $c \in \mathcal{C}$ and any pair of consumers $i_{1} \in \pi_{i_{1}}, i_{2} \in \pi_{i_{2}}$, with $\kappa\left(i_{1}\right)=\kappa\left(i_{2}\right)=c$, and $\tau_{i_{1}}=\tau_{i_{2}}$ it holds that

$$
p \cdot\left(\omega^{0}\left(i_{1}\right)-X\left(i_{1}\right)\right)=p \cdot\left(\omega^{0}\left(i_{2}\right)-X\left(i_{2}\right)\right) .
$$

Proof of Lemma 1. To obtain a contradiction, suppose that 


$$
p \cdot\left(\omega^{0}\left(i_{1}\right)-X\left(i_{1}\right)\right)>p \cdot\left(\omega^{0}\left(i_{2}\right)-X\left(i_{2}\right)\right) .
$$

From Theorem 4 and from the fact that jurisdictions are finite (and thus, of measure zero), there is a consumer $i_{3}$ in another jurisdiction who is identical to $i_{1}$ and, from the equal treatment property of the core, receiving the same utility as $i_{1}$ in the core state of the economy. More formally, there is a consumer $i_{3} \notin \pi_{i_{1}}$ satisfying

$$
\kappa\left(i_{3}\right)=\kappa\left(i_{1}\right), \tau\left(i_{3}\right)=\tau\left(i_{1}\right)
$$

and

$$
u_{\tau\left(i_{3}\right)}\left(X\left(i_{3}\right), Y\left(i_{3}\right), \pi_{i_{3}}\right)=u_{\tau\left(i_{1}\right)}\left(X\left(i_{1}\right), Y\left(i_{1}\right), \pi_{i_{1}}\right) .
$$

Now consider the jurisdiction $G^{*}$ formed by replacing $i_{2}$ with $i_{3}$,

$$
G^{*} \stackrel{\text { def }}{=}\left(\pi_{i_{1}} \cup\left\{i_{3}\right\}\right) \backslash\left\{i_{2}\right\}
$$

Construct the allocation for $G^{*}$ with consumption of public projects equal to $Y(i)$ for each consumer $i \in G^{*}$ and with consumptions of private goods of $X(i)$ for all $i \in G^{*}, i \neq i_{3}$, and with the allocation $x_{i_{3}}=X\left(i_{1}\right)$ - we have simply replaced $i_{2}$ by $i_{3}$ and given $i_{3}$ the same allocation as $i_{1}$. Note that, from (1), the jurisdiction $G^{*}$ can afford this allocation of private goods and the required input of private goods into production and have a surplus of $p \cdot\left(\omega^{0}(i)-X(i)\right)-p \cdot\left(\omega^{0}(j)-X(j)\right)$. From strict monotonicity of preferences for private goods, the membership of $G^{*}$ can afford a bundle of private goods for private consumption and for public project production that would make all members of $G$ better off than they are in the initial state of the economy $(\pi, X, Y, Z, U)$. In particular, each consumer's allocation of all private goods could be increased and the aggregate budget constraint for the jurisdiction $G^{*}$ would still be satisfied. This contradicts the fact that $p$ separates preferred sets from affordable bundles of private goods.

For each crowding type $c$ and any consumer $i$ with crowding type $\kappa(i)=c$ in a jurisdiction with crowding profile $\mathcal{C} \operatorname{pro}\left(\pi_{i}\right)$, define

$$
\psi_{c}\left(Y(i), \mathcal{C} p r o\left(\pi_{i}\right)\right) \stackrel{\text { def }}{=} p \cdot\left(\omega^{0}(i)-X(i)\right) \text {. }
$$


Note that for any two consumers $i$ and $i^{\prime}$ with the same crowding type in the same jurisdiction, from the above argument $p \cdot\left(\omega^{0}(i)-X(i)\right)=p \cdot\left(\omega^{0}\left(i^{\prime}\right)-\right.$ $\left.X\left(i^{\prime}\right)\right)$ (irrespective of their tastes) so $\psi_{c}\left(Y(i), \mathcal{C} \operatorname{pro}\left(\pi_{i}\right)\right)$ is well defined and does not depend on tastes. This is a key result, since it is crucial for the result that prices need not depend on tastes.

It remains to specify public projects and prices for these projects for jurisdictions that do not appear in the core state.

Consider an arbitrary jurisdiction $G \in \mathcal{F}$ and an arbitrary crowding type $c \in \mathcal{C}$ with the property that $\mathcal{C}$ pro $(G)_{c} \neq 0$. Let $(z, y) \in P(\operatorname{pro}(G))$ and suppose that there does not appear a jurisdiction $G^{\prime}$ with $\operatorname{pro}\left(G^{\prime}\right)=\operatorname{pro}(G)$ offering public projects $y$ in the core state of the economy. Now take an arbitrary consumer $i \in G$ of type $c$ (or any consumer $i$ in any jurisdiction $G^{\prime}$ with the same profile) and consider how much he would be willing to pay to join the jurisdiction $G$ offering the public projects bundle $y$. There are two possibilities. (1) It may be that this jurisdiction and public project package are so unattractive that if the consumer were a member of $G$, no amount of income could make him as well off as he is in the core state. (2) There is an amount of income that is feasible for the consumer to pay (his "willingness to pay"), and that leaves him exactly indifferent between $G$ and the jurisdiction to which he is assigned in the core state. From desirability of the endowment for private goods (A.5), we have only these two possibilities. This creates a partition of the set of consumers of crowding type $c$ :

$$
\begin{aligned}
& H_{c}(y, p r o(G)) \stackrel{\text { def }}{=}\left\{i \in N_{c}: \text { for all } x \in \mathbb{R}_{+}^{L},\right. \\
&\left.u_{i}(x, y, \operatorname{pro}(G))<u_{i}\left(X(i), Y(i), \pi_{i}\right)\right\},
\end{aligned}
$$

and

$$
\begin{aligned}
& I_{c}(y, \operatorname{pro}(G)) \stackrel{\text { def }}{=}\left\{i \in N_{c}: \text { there exists } x \in \mathbb{R}_{+}^{L}\right. \text { such that } \\
&\left.u_{i}(x, y, \operatorname{pro}(G))=u_{i}\left(X(i), Y(i), \operatorname{pro}\left(\pi_{i}\right)\right)\right\}
\end{aligned}
$$

Note that if $i$ and $i^{\prime}$ are members of $I_{c}(y, \operatorname{pro}(G))$, while they both have crowding type $c$ their taste types may differ.

For each crowding type $c$, for consumers in the set $I_{c}(y, \operatorname{pro}(G))$ their willingness to pay to join the jurisdiction $G$ is well-defined. Given $c$, define the maximum willingness to pay over all taste types of crowding type $c$ 
represented in the jurisdiction $G$ as follows:

$$
\begin{aligned}
\operatorname{Max} W T P_{c}(y, \operatorname{pro}(G)) \stackrel{\text { def }}{=} \max _{i \in I_{c}(y, p r o(G))} \sup _{x \in \mathbb{R}_{+}^{L}}\left\{p \cdot \omega^{0}(i)-p \cdot x:\right. \\
\left.u_{i}(x, y, \operatorname{pro}(G))=u_{i}\left(X(i), Y(i), \operatorname{pro}\left(\pi_{i}\right)\right)\right\}
\end{aligned}
$$

Given any $\varepsilon>0$, we can think of $\operatorname{MaxWTP}_{c}(y, \operatorname{pro}(G))+\varepsilon$ as a sufficiently high price to discourage all consumers in $I_{c}(y, \operatorname{pro}(G))$ from choosing the package $(y, \operatorname{pro}(G))$ since doing so would make them worse off than in the core state.

To complete the price system, choose $\varepsilon>0$ and let the admission price for any jurisdiction with profile $\operatorname{pro}(G)$ offering public project $y$ be defined as follows:

$$
\psi_{c}(y, \mathcal{C} p r o(G)) \stackrel{\text { def }}{=}\left\{\begin{array}{l}
(a) \frac{-1}{\varepsilon} \text { if } \cup_{t} G_{c t} \subset H_{c}(y, \operatorname{pro}(G)) . \\
(b) \quad M a x W T P_{c}(y, \operatorname{pro}(G)) \text { if } I_{c}(y, p r o(G)) \neq \emptyset .
\end{array}\right\}
$$

Here, if all consumers of crowding type $c$ in $G$ find $G$ very unattractive so much so that no amount of income would make them as well off in $G$ offering the public projects $y$ as in the core state - then the admission price for consumers of crowding type $c$ for this jurisdiction is negative. In the other case, the price is defined so that even those consumers of the taste type that find $G$ and $y$ most attractive are indifferent between $G$ offering $y$ and the core state of the economy. We spell this out in more detail.

Suppose that case (a) obtains. Then except possibly for a set of measure zero, no amount of income is sufficient to induce any consumer of crowding type $c$ to switch to a jurisdiction with profile $\operatorname{pro}(G)$ offering $y$. Therefore, for all $\varepsilon>0$, all consumers are strictly worse off if they join a jurisdiction offering $(y, \mathcal{C}$ pro $(G))$ at the price $-\frac{1}{\varepsilon}$ then they are at the core state.

Suppose instead that case (b) obtains. By the argument above, any consumers who happen to be in the set $H_{c}(y, \operatorname{pro}(G))$ are worse off in the new jurisdiction with the admission price $\psi_{c}(y, \operatorname{pro}(G))$ then they are in their core jurisdictions. By construction, all consumers in $I_{c}(y, \operatorname{pro}(G))$ are no better off if they choose the $G$, offering public projects $y$, with the admission price $\psi_{c}(y, \operatorname{pro}(G))$.

It only remains to show that there exists $\varepsilon>0$ such that for any $z$ satisfying $(z, y) \in P(\mathcal{C}$ pro $(G))$, profits are nonpositive. First note that if for even one $c \in \mathcal{C}$ appearing in $G$, case (a) holds, we can choose $\varepsilon$ arbitrarily 
close to zero, which makes the admission price for type $c$ an arbitrarily large negative number. Obviously then, for small enough $\varepsilon$,

$$
\sum_{\mathcal{C} p r o(G)_{c} \neq 0} n_{c} \psi_{c}(y, \mathcal{C} \operatorname{pro}(G)) \leq p \cdot z \text { for any } z \text { such that }(z, y) \in P(\mathcal{C} p r o(G)) .
$$

Next suppose that case (b) holds for every crowding type represented in $G$ (note this exhausts all possibilities). We proceed by contradiction. Suppose,

$\sum_{\mathcal{C} \operatorname{pro}(G)_{c} \neq 0} n_{c} \psi_{c}(y, \mathcal{C} \operatorname{pro}(G))>p \cdot z$ for some $z$ such that $(z, y) \in P(\mathcal{C}$ pro $(G))$, that is, profits are positive. Then

$\sum_{\mathcal{C} \operatorname{pro}(G)_{c} \neq 0} \operatorname{MaxWTP}_{c}(y, \operatorname{pro}(G))>p \cdot z$ for some $z$ such that $(z, y) \in P(\mathcal{C} \operatorname{pro}(G))$.

In this case, (i) there is enough revenue to cover the costs of producing $y$. Since $I_{c}(y, \operatorname{pro}(G))$ is nonempty, then there exist consumers who are exactly as well off when they join this jurisdiction at the posted prices. Thus, given these prices, the members of the jurisdiction $G$ could (collectively) afford and prefer the jurisdiction $G$ offering the project $y$. This is a contradiction to the separation argument.

Thus, the admission prices constructed above satisfy both the properties that no consumer, given these prices, would strictly prefer jurisdiction and public projects bundle to his core allocation, and profits in all jurisdictions are nonpositive.

Step 4. $(\pi, X, Y, Z, U), p$ and $\psi$ satisfy the requirements for a competitive equilibrium.

First, from the above definition of $\psi_{c}(y, \operatorname{pro}(G))$ it is immediate that in the core state the budget constraint of each consumer is satisfied. From the construction of admission prices, it follows that no consumer $i$ can afford a bundle preferred to $\left(X(i), Y(i), \operatorname{pro}\left(\pi_{i}\right)\right)$ for if he could, then, from monotonicity, there would be an affordable bundle of private goods for the jurisdiction $\pi_{i}$ that would enable all members of $\pi_{i}$ to be better off than they are in the initially given core state of the economy $(\pi, X, Y, Z, U)$. This contradicts the separating hyperplane property of the price system $p$. 
We have already shown zero profits for those jurisdictions in $\pi$ (condition 3.) To prove the impossibility of positive profits (condition 4.), suppose that for some jurisdiction $G \in \mathcal{F}$ and some $(z, y) \in P(\operatorname{pro}(G))$, it holds that

$$
\sum_{i \in G} \psi_{\kappa(i)}(y, \operatorname{pro}(G))-p \cdot z>0 .
$$

But this implies that

$$
\sum_{i \in G}\left(p \cdot \omega^{0}(i)-p \cdot x^{i}\right)-p \cdot z>0
$$

where, for each $i \in G$ it holds that $u_{\tau(i)}\left(x^{i}, y, G\right)=u_{\tau(i)}\left(X(i), Y(i), \pi_{i}\right)$. However, the above expression implies that there are bundles of private goods for each consumer, say $\delta^{i} \in \mathbb{R}_{+}^{L}$ for consumer $i$, so that $\sum_{i \in G}\left(p \cdot \omega^{0}(i)-p\right.$. $\left.x^{i}-p \cdot \delta^{i}\right)-p \cdot z=0$. This contradicts the separating hyperplane property of the price system $p$.

\section{A Appendix B}

Our first theorem requires per capita boundedness (PCB) of utility of finite coalitions in the neighborhood of the population proportions given by the measure, as in Kaneko and Wooders (1986). This assumption simply ensures that equal treatment payoffs are bounded. This assumption is satisfied by most (if not all) economic models where consumers' preferences are described by utility functions.

(PCB) The economy is per capita bounded if there are positive numbers $\delta$ and $K$, with $0<\delta<1$, such that for every finite subset $S \subset N$ and for any feasible state $(\pi, X, Y, Z, U)$ of $S$ with the equal treatment property,

$$
\begin{aligned}
& (1+\delta) \frac{\mu\left(N_{c t}\right)}{\mu(N)} \geq \frac{\left|S_{c t}\right|}{|S|} \geq(1-\delta) \frac{\mu\left(N_{c t}\right)}{\mu(N)} \text { for all }(c, t) \in \mathcal{C} \times \mathcal{T} \\
& \Rightarrow U(i) \leq K \text { for all } i \in S .
\end{aligned}
$$

Our next Proposition shows that strict small group effectiveness implies per capita boundedness. 
Proposition. Assume (A.2), then (A.4), SSGE, implies PCB.

\section{Proof of Proposition}

The proof is similar to the one in Kaneko and Wooders (1986, Lemma 3.3). Suppose the negation. Then, there exists an increasing sequence $\left\{K^{\nu}\right\}$ such that for some $\delta \in[0,1]$ for some $c_{0}, t_{0}$ one could choose sequences of subsets $\left\{S^{\nu}\right\}$ of consumers and feasible states of the economy $\left\{\left(\pi^{\nu}, X^{\nu}, Y^{\nu}, Z^{\nu}, U^{\nu}\right)\right\}$ with the equal treatment property, where, for each $\nu$, $\left(\pi^{\nu}, X^{\nu}, Y^{\nu}, Z^{\nu}, U^{\nu}\right)$ is relative to $S^{\nu}$ such that

$$
\begin{aligned}
& (1+\delta) \frac{\mu\left(N_{c t}\right)}{\mu(N)} \geq \frac{\left|S_{c t}^{\nu}\right|}{\left|S^{\nu}\right|} \geq(1-\delta) \frac{\mu\left(N_{c t}\right)}{\mu(N)} \text { for all }(c, t) \in \mathcal{C} \times \mathcal{T} \\
& \Rightarrow u_{t_{0}}\left(X^{\nu}(i), Y^{\nu}(i), \operatorname{pro}\left(\pi_{i}^{\nu}\right)\right)>K^{\nu} \text { for all } i \in S_{c_{0} t_{0}} .
\end{aligned}
$$

From assumptions (A.2) - boundedness of inputs implies boundedness of public project outputs, and (A.4), SSGE, we can find an $\nu_{0}$ such that for all consumers $i$ of type $\left(c_{0}, t_{0}\right)$ in $S^{\nu}$ and for some private good, say the $\ell^{t h}$, it holds that

$$
\begin{gathered}
U^{\nu}(i)=u_{t_{0}}\left(X^{\nu_{0}}(i), Y^{\nu_{0}}(i), \operatorname{pro}\left(\pi_{i}^{\nu_{0}}\right)\right)>K^{\nu_{0}} \\
\Rightarrow X^{\nu_{0}}(i)_{\ell}+Z^{\nu}(i)_{\ell}>\frac{\mu(N)}{(1-\delta) \cdot \mu\left(N_{c_{0} t_{0}}\right)} \Sigma_{c, t} \omega^{0}(c t)_{\ell} ;
\end{gathered}
$$

to have much utility, one must have much of at least one private good to consume and/or to use in production of public goods. Since consumptions of private goods and inputs of private goods into the production of public goods are all non-negative, it follows that:

$$
\begin{aligned}
& \sum_{i \in S^{\nu_{0}}}\left(X^{\nu_{0}}(i)+z^{\nu_{0}}(i)\right)_{\ell} \\
& >\left|S_{c_{0} t_{0}}^{\nu}\right| \frac{\mu(N)}{(1-\delta) \cdot \mu\left(N_{\left.c_{0} t_{0}\right)}\right)} \Sigma_{c, t} \omega^{0}(c t)_{\ell} \\
& \geq\left|S^{\nu}\right| \Sigma_{c, t} \omega^{0}(c t)_{\ell}\left(\text { since, from } 2, \frac{\left|S_{c t}^{\nu}\right|}{\left|S^{\nu}\right|} \geq(1-\delta) \frac{\mu\left(N_{c t}\right)}{\mu(N)}\right) \\
& \geq \Sigma_{c, t}\left|S_{c, t}^{\nu}\right| \omega^{0}(c t)_{\ell} \\
& =\Sigma_{i \in S} \omega^{0}(i)_{\ell} .
\end{aligned}
$$

This contradicts the feasibility of the allocation.

Given the measure space of consumers $N$, a characteristic function game $V$ without side payments is a correspondence on $\mathcal{F}$ which assigns to each coalition $S \in \mathcal{F}$ a subset $V(S)$ with the following properties:

1. $V(S)$ is a nonempty closed subset of $\mathbb{R}^{S}$ for all $S \in \mathcal{F}$; 
2. $V(S) \times V(W) \subset V(S \cup W)$ for any $S, W \in \mathcal{F}$ with $S \cap W=\emptyset$;

3. $\inf _{i \in N} \sup V(\{i\})>-\infty$;

4. for any $S \in \mathcal{F}, V(S) / \cup\left[\right.$ interior $\left.V(\{i\}) \times \mathbb{R}^{S-\{i\}}\right]$ is nonempty and bounded.

A characteristic function game can be generated by an economy in the usual way. Specifically, given a finite coalition $S$, define

$V(S) \stackrel{\text { def }}{=}\left\{\bar{u} \in \mathbb{R}^{S}:\right.$ for some feasible state of the economy for $S$, say $(\pi, X, Y, Z, U)$, it holds that for each $i \in S, u_{\tau(i)}\left(X(i), Y(i), \mathcal{C}\right.$ pro $\left.\left.\left(\pi_{i}\right)\right) \geq \bar{u}_{i}\right\}$.

Condition 1 is satisfied for the game derived from the economy from closeness of the consumption sets $\mathcal{X}_{c t}$, continuity of utility functions, and closeness of the production possibility sets. Condition 2 . is immediate since one possibility open to a coalition (or a jurisdiction) consisting of $S \cup W,(S \cap W=\emptyset)$ is to form a partition into disjoint coalitions $S$ and $W$. Condition 3 . is also immediate since there are only a finite number of types and since the supremum of a finite set of real numbers is a real number. Condition 4 is simply that the set of feasible and individually rational payoffs is bounded above. This follows from the assumptions that a finite amount of private goods can produce only a bounded amount of public and private goods (A.2). Kaneko and Wooders also require that collection of finite games satisfies per capita boundedness, which follows from our assumptions.

One additional definition is required. A function $h: N \rightarrow \mathbb{R}_{+}$is in the equal treatment core of the game if it is feasible and if (a) for all $i, j \in N$, whenever $\kappa(i)=\kappa(j)$ and $\tau(i)=\tau(j)$ it holds that $h(i)=h(j)$ and (b) for all finite coalitions $S \subset N$ it holds that $\left.h\right|_{S} \notin \operatorname{int} V(S)$ where $\left.h\right|_{S}$ is the restriction of $h$ to the membership of $S$. (Since $N$ consists of a finite number of types of consumers and a positive measure of consumers of each type we need not allow for exceptional sets of consumers of measure zero.)

Theorem 1. Nonemptiness of the equal-treatment core. Under assumptions (A.2), and (A.4), the equal-treatment core of the game generated by the economy is nonempty.

Proof of Theorem 1. The proof of this Theorem follows by showing that the conditions required for Kaneko and Wooders (1986, Theorem 1), demonstrating the nonemptiness of the $f$-core of a continuum game, are 
satisfied by the game induced by the economy. First, we will review the model and statement of Theorem 1 of Kaneko and Wooders (1986).

The nonemptiness theorem stated in Kaneko and Wooders (1986) does not mention the equal-treatment property. Their result is proven, however, by showing existence of an equal-treatment $f$-core payoff that is the limit of equal-treatment payoffs in finite approximating games. We will briefly sketch the result.

Wooders (1983) shows that sequences of games with types satisfying per capita boundedness have nonempty approximate cores. This result is based on the result that, in large games, when all "improvement" can be carried out by coalitions bounded in size and the bound is small relative to the economy, provided payoff sets do not contain segments parallel to the axes - called strong comprehensiveness or nonlevelness- then all payoffs in the core of a (finite) game have the equal treatment property. Moreover, even without strong comprehensiveness, under these conditions the core, when nonempty, contains an equal-treatment payoff. Building on these results, Shubik and Wooders (1983) establish that for any sequence of games satisfying per capita boundedness, eventually there are equal treatment payoffs in approximate cores. Kaneko and Wooders (1986) use this result to show that there is a sequence of vectors $\left\{u^{\nu}\right\}$, where $u \in \mathbb{R}^{C T}$ (taking $C T$ as the number of types of consumers) represents an equal treatment payoff in the core of a finite game with proportions of consumers in the finite games converging to the proportions in the continuum limit game. From per capita boundedness, the sequence $\left\{u^{\nu}\right\}$ has a converging subsequence, converging to, say $u^{*}$. The

function $h: N \rightarrow \mathbb{R}$ defined by $h(i) \stackrel{\text { def }}{=} u_{c t}^{*}$ when $i$ is of type $(c, t)$ is in the core of a continuum limit game.

\section{References}

[1] Allouch, N. and M. Wooders (2008) "Price taking equilibrium in club economies with multiple memberships and unbounded club sizes," Journal of Economic Theory 140, 246-278.

[2] Aumann R.J. (1964) "Markets with a continuum of traders," Econometrica $32,39-50$. 
[3] Berglas, E. and D. Pines (1981) "Clubs, local public goods and transportation models; A synthesis," Journal of Public Economics 15, 141162.

[4] Bewley, T. (1981) "A critique of Tiebout's theory of local public expenditure;" Econometrica 49, 713-740.

[5] Cole, H. and E. Prescott (1997) "Valuation equilibria with Clubs" Journal of Economic Theory 74, 19-39.

[6] Conley, J., and H. Konishi (2002), "The Tiebout theorem: On the existence of asymptotically efficient migration-proof equilibria," Journal of Public Economics 86, 243-62.

[7] Conley, J. and M.H. Wooders (1997) "Equivalence of the core and competitive equilibrium in a Tiebout economy with crowding types," Journal of Urban Economics 41, 421-440.

[8] Demange G. (1994) "Intermediate preferences and stable coalition structures," Journal of Mathematical Economics, 1994, 45-48.

[9] Dunz, K. (1989) "Some comments on majority rule equilibrium in local public goods economies," Journal of Economics Theory, 47, 228-34.

[10] Debreu G. and H. Scarf (1963) "A limit theorem on the core of an economy" International Economic Review 4, 235-246.

[11] Ellickson, B. (1979) "Competitive equilibrium with local public goods" Journal of Economic Theory 21, 46-61.

[12] Ellickson, B., B. Grodal, S. Scotchmer, and W. Zame (1999) "Clubs and the market" Econometrica 67, 1185-1218.

[13] Epple, D., R. Filimon and T. Romer, (1984) "Equilibrium among local jurisdictions: Toward an integrated treatment of voting and residential choice," Journal of Public Economics 24, 281-308.

[14] Epple, D., R. Filimon and T. Romer, (1993) "Existence of voting and housing equilibrium in a system of communities with property taxes," Regional Science and Urban Economics 23, 585-610. 
[15] Foley, D. (1970) "Lindahl's Solution and the core of an economy with public goods," Econometrica 38, 66-72.

[16] Gravel, N. and S. Thoron (2007) "Does endogenous formation of jurisdictions lead to wealth stratification," Journal of Economic Theory 132, 569-583.

[17] Greenberg, J. and B. Shitovitz (1988) "Consistent voting rules for competitive local public good economies," Journal of Economic Theory 46, 223-36.

[18] Greenberg, J. and S. Weber (1986) "Strong Tiebout equilibrium under restricted preference domain," Journal of Economic Theory 38, 101-11.

[19] Guesnerie, R. and C. Oddou (1981) "Second best taxation as a game," Journal of Economic Theory 25, 67-91.

[20] Hamilton, B.W. (1975), "Zoning and property taxation in a system of local governments," Urban Studies 12, 205-211.

[21] Hammond, P., M. Kaneko and M. H. Wooders (1989) "Continuum economies with finite coalitions: Core, equilibria, and widespread externalities," Journal of Economic Theory 49, 113-134.

[22] Hildenbrand, W. (1974) "Core and equilibria of a large economy," Princeton University Press.

[23] Kaneko, M. and Wooders, M.H. (1986) "The core of a game with a continuum of consumers and finite coalitions: The model and some results," Mathematical Social Sciences 12, 105-137.

[24] Kaneko, M. and Wooders, M.H. (1989) "The core of a continuum economy with widespread externalities and finite coalitions: From finite to continuum economics," Journal of Economic Theory 49, 135-168.

[25] Kaneko, M. and Wooders, M.H. (1996) "The nonemptiness of the fcore of a game without side payments," International Journal of Game Theory 25 (1996), 245-258.

[26] Keiding, H. (1976) "Cores and equilibria in an infinite economy," in Computing Equilibrium: How and Why, J. Los and M.W. Los, eds. North Holland, Amsterdam/Oxford/New Tork, p. 65-73. 
[27] Konishi, H. (2006) "Tiebout's tale in spatial economies; Entrepreneurship, self-selection and efficiency," Presented at the North American Regional Science Council Conference, Toronto, November 2006, Regional Science and Urban Economics (2008), doi: 10.1016/j.regsciurbeco.2008.05.010

[28] Konishi, H. (1996) "Voting with ballots and feet: Existence of equilibrium in a local public good economy," Journal of Economic Theory 68, 480-509.

[29] Konishi, H., M. Le Breton, and S. Weber (1998) "Equilibrium in a finite local public goods economy," Journal of Economic Theory 79, 224-244.

[30] Kovalenkov, A. and M. H. Wooders (2003) "Approximate cores of games and economies with clubs," Journal of Economic Theory 110, 87-120.

[31] Manning, J. (1992) "Efficiency of economies with jurisdictions and local public projects," typescript, Department of Economics, University of Rochester.

[32] Mas-Colell, A. (1980) "Efficiency and decentralization in the pure theory of public goods," Quarterly Journal of Economics 94, 625-641.

[33] McGuire, M. (1974) "Group segregation and optimal jurisdictions," Journal of Political Economy, 82, 112-132.

[34] Nechyba, T. (1997) "Existence of equilibrium and stratification in local and hierarchical Tiebout economies with property taxes and voting," Economic Theory, 10, 277-304.

[35] Novshek, W. and H. Sonnenschein (1982) "Small efficient scale as a foundation for Walrasian equilibrium," Journal of Economic Theory, 22, pp. 243-255, April 1980.

[36] Pauly, M. (1970) "Cores and Clubs", Public Choice 9, 53-65.

[37] Rose-Ackerman, S. (1979) "Market of models of local government, exit, voting and the land market," Journal of Urban Economics 6 319-37.

[38] Shubik, M. (1959) "Edgeworth market games', in Luce, F.R. and Tucker, A.W. (eds.), Contributions to the Theory of Games IV, Annals of Mathematical Studies 40, Princeton: Princeton University Press, 277-278. 
[39] Shubik, M. and Wooders, M.H. (1982) "Near-markets and marketgames," Cowles Foundation Discussion Paper. Published in part in Economic Studies Quarterly (1986) 37, 289-299 and in full ias "Clubs, near markets and market games, in Topics in Mathematical Economics and Game Theory; Essays in Honor of Robert J. Aumann, eds. M. H. Wooders American Mathematical Society Fields Communication Volume.

[40] Tiebout, C. (1956) "A pure theory of local expenditures," Journal of Political Economy 64, 416-424.

[41] Vohra, R. (1984) "Local public goods and average cost pricing" Journal of Mathematical Economics 13, 51-67.

[42] Vohra, R. (1987) "Local public goods as indivisible commodities," Regional Science and Urban Economics 17, 191-208.

[43] Weber, S. and S. Zamir (1985) "Existence of equilibrium in economies with a local public good," Journal of Economic Theory 35, 178-85

[44] Westhoff, F. (1977) "Existence of equilibrium in economies with a local public good," Journal of Economic Theory 14, 84-112.

[45] Winter, E. and M. Wooders (1994) "An axiomatization of the core of finite games and continuum games with finite coalitions," Social Choice and Welfare 11:165-175.

[46] Wooders M.H. (1978) "Equilibria, the core, and jurisdiction structures in economies with a local public good," Journal of Economic Theory 18, 328-348.

[47] Wooders, M.H. (1980) "The Tiebout Hypothesis: Near optimality in local public good economies," Econometrica 48, 1467-1486.

[48] Wooders, M.H (1983) "The epsilon core of a large replica game," Journal of Mathematical Economics, 11, 277-300.

[49] Wooders, M.H. (1985) "A Tiebout Theorem," Institute for Mathematics and its Applications, University of Minnesota Preprint \#128, published in Mathematical Social Sciences 18 (1989), 33-55.

[50] Wooders, M.H. (1994) "Equivalence of games and markets," Econometrica $62,1141-1160$. 
[51] Wooders, M.H. (1997) "Equivalence of Lindahl equilibria with participation prices and the core," Economic Theory 9, 113-127. 